\title{
Bacterial "galactosemia" is caused by cytoplasmic interference of an essential cell wall biosynthesis glycosyltransferase
}

Cameron Habib ${ }^{\text {a }}$, Anna Mueller³, Ming Liư,

1. Department of Biology, Northeastern University, Boston, MA 02150, USA

2. Department of Molecular and Cell Biology, Harvard University, Cambridge, MA 02138, USA

3. Institute for Pharmaceutical Microbiology, University Hospital Bonn, University of Bonn, 53115 Bonn, Germany

4. Department of Microbiology, Nanjing Agricultural University, Nanjing, 210095, China

5. Department of Microbiology, Perelman School of Medicine, University of Pennsylvania, Philadelphia, PA, 19104, USA

a Current address: Department of Microbiology, Harvard Medical School,

$23 \quad * \quad$ For correspondence, please address to y.chai@northeastern.edu; Boston, MA, USA. yingjiesun@g.harvard.edu 


\section{Abstract}

Bacterial galactosemia or "galactose death," triggered by incomplete galactose

27 metabolism, was first discovered in Escherichia coli and Salmonella six decades ago,

28 and later in many other microorganisms, yet the mechanism for the toxicity and

29 subsequent cell death remains unclear. In Bacillus subtilis, galactosemia is manifested

30 by a buildup of uridine-diphosphate-galactose (UDP-Gal) and a strong toxicity

31 phenotype characterized by cell shape abnormality and rapid cell lysis. Here we present

32 evidence that in $B$. subtilis, the toxicity is due to inhibition of cell wall biosynthesis

33 through interference of the essential glycosyltransferase MurG that carries out lipid II

34 synthesis from lipid I and uridine-diphosphate- $N$-acetyl-glucosamine (UDP-GIcNAc).

35 Single-molecule imaging reveals real-time inhibition of cell wall biosynthesis and MurG

36 activities in cells exhibiting toxicity. We further show that in vitro, MurG is able to utilize

37 UDP-Gal as a substrate generating a "toxic" lipid II, causing a potential poisoning effect

38 on peptidoglycan crosslinking. Evidence also suggests a similar mechanism in Vibrio

39 cholerae and Staphylococcus aureus. Lastly, a strong synergistic lethality was seen in

40 S. aureus wild-type cells treated with both galactose and sub-lethal doses of cell-wall

41 antibiotics. Our study provides mechanistic explanation of the toxicity associated with

42 bacterial galactosemia and its potential application in antibacterial solutions. 


\section{Significance}

46 Galactosemia is a potentially fatal genetic disorder due to incomplete galactose

47 metabolism, found in both eukaryotic and prokaryotic organisms. The molecular

48 mechanisms of galactosemia-associated toxicity remain unclear in all cases. Here we

49 present evidence that in the bacterium Bacillus subtilis, the toxicity is due to interference

50 of an essential glycosyltransferase, MurG, which concerts lipid I to lipid II during

51 peptidoglycan biosynthesis, by a nucleotide sugar derived from galactose metabolism.

52 This interference leads to a halt of cell wall biosynthesis and structural defects causing

53 rapid cell lysis. Our evidence also suggests a similar mechanism in other bacteria such

54 as Staphylococcus aureus and Vibrio cholerae. Our study may help solve the long-time

55 puzzle of bacterial galactosemia first uncovered six decades ago. 


\section{Introduction}

Galactosemia arises from mutation or deficiency in the near ubiquitous Leloir pathway ${ }^{1}$. This pathway, conserved in both prokaryotes and eukaryotes, consists of three enzymes (GalK, GalT, and GalE) and is responsible for the metabolism of a-Dgalactose to glucose-1-phosphate (Fig. 1A). The metabolic process begins as galactokinase (GalK) converts $\alpha-D$-galactose to galactose-1-phosphate (Gal-1-P), followed by galactose-1-P-uridylyltransferase (GalT) converting Gal-1-P and uridinediphosphate-glucose (UDP-GIc) to uridine-diphosphate-galactose (UDP-Gal) and glucose-1-P. Lastly, UDP-Gal is converted back to UDP-Glc by UDP-galactose 4-

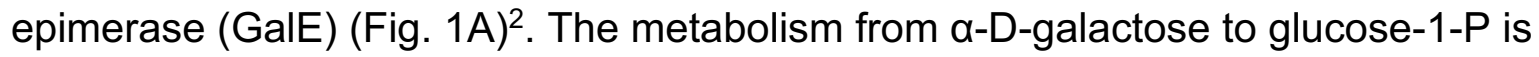
believed to be self-sustainable by the activities of these three enzymes ${ }^{1}$. In bacteria, the inability to completely metabolize galactose causes a strong toxicity phenotype, a phenomenon phrased as "galactose death" or "bacterial galactosemia," first described in E. coli and Salmonella in $1959^{3,4}$ and later observed in many other bacteria ${ }^{5,6}$, with the underlying mechanism still unknown.

In Bacillus subtilis, a deletion in the galE gene causes a buildup of UDP-Gal when cells grow in excess galactose and a strong toxicity phenotype characterized by cell shape deformation and rapid cell lysis ${ }^{6,7}$. While buildup of UDP-Gal causes toxicity, this nucleotide sugar is needed for a number of important processes, including synthesis of an exopolysaccharide (EPS) for biofilm formation in B. subtilis ${ }^{7,8}$. Therefore, producing UDP-Gal but maintaining its cellular level below a threshold that triggers toxicity must be an important regulation during galactose metabolism. In a previous work, we uncovered a shunt mechanism in B. subtilis allowing bypass of the toxicity upon strong biofilm induction when UDP-Gal was used as a precursor for EPS biosynthesis and exported in large quantity ${ }^{7}$. Galactose can be naturally and abundantly available to $B$. subtilis when this soil bacterium utilizes complex carbohydrates available in the environment such as plant-derived galactan ${ }^{9}$. B. subtilis possesses a dedicated pathway for uptake and hydrolysis of galactan into galactose for metabolic purposes as well as to make EPS for biofilm formation. Biofilms in turn play a key role in establishing intimate relationships between $B$. subtilis and the plant ${ }^{7,8,10-13}$. 
Bacterial cell wall consists of cross-linked peptidoglycan chains, whose building block is delivered via a membrane lipid carrier, lipid II. Synthesis of lipid II is carried out in the cytoplasm by an essential glycosyltransferase MurG, through the addition of $N$ -

91 acetyl-glucosamine (GIcNAc) to lipid I, by utilizing a nucleotide sugar UDP-N-acetyl-

92 glucosamine (UDP-GlcNAc) ${ }^{14,15}$. Once synthesized, lipid II is flipped across the

93 cytoplasmic membrane by a flippase, and the sugar-peptide building block polymerized

94 into full-length peptidoglycan by extracellular penicillin-binding proteins ${ }^{16-18}$. The

95 biosynthetic machinery responsible for peptidoglycan polymerization in both $E$. coli and

96 B. subtilis has been shown to be associated with MreB filaments, as they move around

97 the cell, a circumferential movement driven by, and directly correlated to, peptidoglycan

98 synthesis ${ }^{19,20}$. Using fluorescent protein fusions to MreB, the movement of MreB

99 filaments can be visualized under Total Internal Reflection Fluorescence (TIRF)

100 microscopy and has been used as an assay for measuring dynamic cell wall

101 biosynthesis ${ }^{21}$.

102

In this study, we investigated the mechanism of cell toxicity associated with

104 bacterial galactosemia in B. subtilis. Our evidence suggests a strong link between the

105 toxicity and inhibition of cell wall biosynthesis, with the essential glycosyltransferase

106 MurG and lipid precursor biosynthesis as targets.

107

108 Results

109 Galactose triggers cell shape abnormality and rapid cell lysis in a $B$. subtilis $110 \Delta$ galE mutant.

111 B. subtilis $\Delta$ galE cells quickly lyse when grown in the presence of galactose ${ }^{7}$.

112 Evidence suggests that this toxicity is due to accumulation of UDP-Gal as a result of

113 incomplete galactose metabolism (Fig. 1A) . To further characterize the toxicity, $\Delta g a l E$

114 cells were grown in LB supplemented with galactose $(0.5 \%$, w/v), periodically collected,

115 stained with live/dead fluorescent dyes, and imaged under fluorescent microscopy. We

116 found that cell optical density $\left(\mathrm{OD}_{600}\right)$ of the culture peaked at around 0.3 , and an hour

117 past the peak, the ratio of dead versus total cells increased exponentially to

118 approximately $98 \%$ based on live/dead cell staining (Fig. 1B). In the absence of 
119 galactose, this number was approximately 1\%. Phase contrast imaging showed gross

120 structural deformities of cells such as rounding out of the regular bacilli shape, cell wall

121 bulges, and cell lysis not seen in untreated cells (indicated by arrows, Fig. 1C).

122 Scanning electronic microscopy showed clear damage in cell structural integrity, with

123 deformations of cell shape and apparent defects in the cell envelope (Fig. 1D). To

124 quantitatively analyze how individual cells were impacted, the maximum cell width was

125 determined by single cell analysis under microscopy and the distribution plotted. 90 min

126 after addition of galactose, the average width distribution of the galactose-treated cells

127 increased $\sim 20 \%$ over that of untreated cells (Fig. 1E), indicating that both the ratio and

128 the severity of cells exhibiting toxicity increased over time. In the same assays without

129 addition of galactose, the doubling time of $\Delta$ galE cells did not differ from that of the WT

130 (Fig. S1A).

\section{Production, not depletion, of UDP-glucose, contributes to toxicity.}

134 galactose metabolism (Fig. 1A). It is also a common precursor for syntheses of complex

135 polysaccharides such as teichoic acids, lipopolysaccharides, and a signal for cell size

136 control in bacteria ${ }^{22,23}$. Given the importance of UDP-Glc, it was previously proposed

137 that the toxicity associated with incomplete galactose metabolism was due to depletion

138 of UDP-Glc, rather than accumulation of UDP-Gal ${ }^{24}$. Depletion of UDP-Glc can occur

139 when the uridine-di-phosphate group is transferred from UDP-Glc to Gal-1-P to form

140 UDP-Gal and Glu-1-P (Fig. 1A). In cells lacking GalE, regeneration of UDP-Glc from

141 UDP-Gal is blocked, thus gradually depleting UDP-Glc while accumulating UDP-Gal ${ }^{6}$.

142 In the absence of active galactose metabolism, the primary source of UDP-Glc is

143 believed to be made from Glc-1-P by a UDP-glucose pyrophosphorylase (GtaB, Fig.

$1441 \mathrm{~A})^{22,25}$. In B. subtilis, loss of GtaB activity leads to depletion of UDP-Glc ${ }^{25}$. To assess

145 the impact of UDP-Glc depletion in B. subtilis, a gtaB deletion strain was created and

146 cells were grown in LB without addition of galactose. No clear growth defect or toxicity

147 was observed (Fig. 2A). Although not essential for growth, the $\Delta g t a B$ mutant was found

148 completely deficient in biofilm formation (Fig. S1B). We previously showed that the EPS

149 of the $B$. subtilis biofilm matrix was rich in glucose ${ }^{7}$. The biofilm deficiency of the $\Delta g t_{a} B$ 
150 mutant suggests that UDP-Glc is the precursor for providing the glucose moiety of the

151 EPS. It also confirms that GtaB activity is the primary source of UDP-Glc synthesis in $B$.

152 subtilis.

153

Upon further investigation, we found that not only does UDP-Glc depletion have no impact on cell viability, but production of UDP-Glc by GtaB is a prerequisite for accumulating UDP-Gal and causing toxicity during galactose metabolism. A double

157 mutant of $\Delta g a l E \Delta g t a B$ with an IPTG-inducible copy of gtaB was constructed. The 158 engineered strain was grown in LB with galactose and varying amounts of IPTG to 159 induce gtaB expression. The observed toxicity, judged by growth curve, correlated well 160 with gtaB induction in a dose dependent manner (Fig. 2B). These findings suggest that 161 continuous production of UDP-Glc by GtaB is necessary for accumulating UDP-Gal and 162 triggering toxicity in the $\Delta$ galE cells (Fig. 1A).

\section{Toxicity is associated with inhibition in cell wall biosynthesis.}

Given the cell shape abnormality observed in the $\Delta$ galE cells and their resemblance to known cell shape phenotypes caused by defects in cell wall biosynthesis ${ }^{26}$, we sought to test if the toxicity is linked to impaired cell wall

168 biosynthesis. Cell wall biosynthetic machinery has been shown to be bound to and pull 169 along MreB filaments as cargo ${ }^{19,21,27}$, a motion driven by cell wall synthesis. Inhibition

170 of cell wall synthesis via cell wall targeting antibiotics can causes MreB movement to

171 cease ${ }^{19,28}$. To determine in real-time if the dynamics of cell wall biosynthesis were

172 altered in cells exhibiting toxicity, a MreB-mNeonGreen fusion was expressed in the $B$.

173 subtilis $\Delta$ galE mutant. Cells were immobilized under a $\mathrm{CH}$ agar pad with galactose

$174(0.5 \%, w / v)$ and the activity of the MreB filaments observed using Total Internal

175 Reflection Fluorescence (TIRF) microscopy with imaging every 30 minutes. Movement

176 of the fluorescent foci in single cells over the course of the time-lapse imaging was

177 calculated and plotted (Fig. 2C, D, and Supple. Movie 1). The results indicate that

178 movement of the MreB filaments decreased dramatically over time, with movement

179 ceasing and filaments depolymerizing completely $\sim 60$ minutes after galactose addition 
180 (Fig. 2C, D). These results suggest that cell wall biosynthesis was blocked in the $\Delta$ galE 181 cells exhibiting toxicity.

Toxicity is not likely due to inhibition in biosynthesis of UDP-GIcNAc. fructose-6-phosphate (Fru-6-P) to glucosamine-6-phosphate (GlcN-6-P) by a glutaminefructose-6-phosphate aminotransferase (GImS, Fig. 3A). A recent study showed a similar cell shape deformation and lysis phenotype, as seen in $\Delta g a l E$, in a $B$. subtilis $\Delta g I m R$ mutant ${ }^{29}$. glmR encodes a regulatory protein for the aminotransferase GImS, which carries out the first step in the synthesis of UDP-GIcNAc (Fig. 3A). When the cellular UDP-GIcNAc levels are low, GImR activates GImS, thus promoting synthesis of UDP-GIcNAc. In the presence of excess UDP-GIcNAc, GImR is inhibited upon binding directly to UDP-GIcNAc. This serves as a checkpoint mechanism for UDP-GIcNAc biosynthesis (Fig. 3A $)^{29}$. hypothesized that if UDP-Gal targets any of these enzymes, it can cause toxicity. For example, it is possible that UDP-Gal directly binds to and inhibits GImR and thus blocks UDP-GIcNAc biosynthesis given that GImR also binds to UDP-Glc, which is structurally similar to UDP-Gal ${ }^{30}$. If so, providing GImR in excess could partially alleviate toxicity.

200 We took a genetic approach to test this by overexpressing glmR in the $\Delta$ galE mutant. 201 However, no toxicity rescue was observed (Fig. 3B). glmS was similarly overexpressed

202 since it was shown that the cell shape deformation and lysis phenotype of $\Delta g \operatorname{lm} R$ can 203 be rescued by overexpression of $\mathrm{g} / \mathrm{mS}^{29}$. Again, no rescue in toxicity was observed

204 (Fig. 3B). Finally, we overexpressed glmU and glmM, respectively, and none showed 205 toxicity rescue (Fig. 3B). Thus, our genetic evidence suggests that biosynthesis of UDP206 GlcNAc, the initial cytoplasmic stage in peptidoglycan biosynthesis, is likely not the 207 target associated with toxicity. 
The final step in lipid II biosynthesis is carried out by the essential glycosyltransferase MurG, through the addition of $N$-acetyl-glucosamine to lipid I (Fig.

212 3A). To test if MurG is a possible target, we introduced an IPTG inducible copy of murG

213 into the $B$. subtilis $\Delta$ galE mutant. The resulting strain was grown in the presence of both

214 galactose and IPTG. Upon addition of $200 \mu \mathrm{M}$ IPTG, there was a significant reduction of

215 toxicity (as judged by the growth curve) compared to no IPTG addition (Fig. 3C). Note

216 that a slight toxicity reduction was seen even in the absence of IPTG, likely due to leaky

217 expression of murG from the hyperspank promoter. This suggests that murG

218 overexpression can partially rescue the toxicity phenotype in $B$. subtilis. This rescue

219 was unique to MurG, as other essential enzymes $m n a A$, tagB, and murA $A$, also

220 involved in the utilization of UDP-GIcNAc, did not show any rescue upon similar

221 overexpression (Fig. S1C).

We further assayed the toxicity rescue by murG overexpression by analyzing the growth of single cells. The $\Delta$ galE cells with the inducible murG were grown on a $\mathrm{CH}$

225 agar pad containing galactose $(0.5 \%, \mathrm{w} / \mathrm{v})$ and varying concentrations of IPTG and 226 imaged to determine the doubling time of individual cells. Strong murG expression

227 (addition of $500 \mu \mathrm{M}$ IPTG) caused cells to regain close to the WT growth rates (Fig. 3D).

228 Further, cells were found to maintain a consistent max cell width for a greater period of

229 time as compared to without MurG overexpression (Fig. S1D). As the control, murG 230 overexpression did not result in an increased growth rate in the absence of galactose

231 (Fig. S1A, S1E). To determine if murG overexpression indeed relieved inhibition of cell

232 wall biosynthesis, the mreB-mNeonGreen construct previously utilized was introduced

233 into the above murG inducible strain and the resulting strain was similarly imaged for

234 MreB-mNeonGreen movement and filamentation. Upon murG overexpression, the 235 movement and number of MreB filaments increased significantly above those in $\Delta g a l E$ 236 cells, at 60 and even 90 min after addition of galactose (Fig. 2E-F, compare Fig. 2D and

237 2F; Supple. Movie 2). Taken together, this suggests that MurG activity may be the 238 target or cause of UDP-Gal-mediated toxicity. Lastly, overexpression of neither MurAA, 239 the first committed step in peptidoglycan synthesis ${ }^{31}$, nor MraY, the first membrane 240 step of peptidoglycan synthesis in which lipid I is generated ${ }^{32}$, resulted in rescue on 
241 blocked cell wall biosynthesis as observed upon overexpression of murG (Supple.

242 Movie 3).

In vivo evidence for inhibition of peptidoglycan precursor synthesis and MurG in cells exhibiting toxicity.

Inhibition of MurG or subsequent cell wall biosynthesis step can cause an accumulation of lipid precursors, and ultimately halt of cell wall biosynthesis ${ }^{15,33,34}$, which can be measured using an established assay ${ }^{35}$, To test this, $\Delta$ galE cells were grown in LB in the presence or absence of galactose and collected every 30 minutes. Lipids were extracted, labeled with biotin-D-Lys, and detected by immunoblotting. In cells grown in the absence of galactose (lanes with Gal “-”, Fig. 4A-B), accumulation of peptidoglycan precursor was not detected, likely due to their continued use in peptidoglycan synthesis. However, in the presence of galactose, cells accumulated lipid precursors in concentrations easily detected (lanes with Gal "+”, Fig. 4A-B), indicating an inhibition of use of these intermediates. As a positive control, when cells were treated with vancomycin, inhibiting peptidoglycan cross-linking, it resulted in an even stronger accumulation of lipid precursors over untreated cells (Vanc, Fig. 4A-B). Note that in this assay, we did not distinguish whether it is accumulation of lipid I or lipid II.

To further examine the impact of toxicity on MurG in vivo, a HALO-MurG fusion protein was created in the $\Delta g a l E$ background and engineered cells were imaged by TIRF microscopy. In the absence of galactose, MurG was found to freely diffuse

264 Teixo, Fig. S2A and Supple. Movie 4). The newly discovered antibiotic teixobactin was

265 shown to bind to undecaprenyl-coupled lipid intermediates and thus block cell wall

266 biosynthesis in Gram-positive bacteria ${ }^{36}$. We first tested if and how this novel antibiotic

267 may impact MurG activities in vivo. Upon addition of teixobactin $(2.5 \mu \mathrm{g} / \mathrm{mL})$, free

268 diffusion of HALO-MurG was largely inhibited (+Teixo, Fig. S2A and Supple. Movie 4).

269 As an additional control, the directional movement of MreB filaments was similarly

270 inhibited upon addition of teixobactin (Fig. S2B and Supple. Movie 5). These results

271 suggest that the diffusive state of MurG may be used as an assay for its enzymatic 
272 activity. By using this system, we next determined the effect of UDP-Gal accumulation

273 on MurG activities, $0.5 \%$ galactose was added to the media and imaging of HALO-MurG

274 performed. By 120 minutes after the addition of galactose, diffusion of HALO-MurG was

275 found to be largely inhibited, further showing that accumulation of UDP-Gal causes an

276 inhibition of MurG activity in vivo (Supple. Movie 6).

UDP-Gal causes production of a "toxic" lipid II in vitro.

The above evidence suggests that accumulation of UDP-Gal interferes with the

280 function of MurG in vivo. To further test this idea in vitro, B. subtilis MurG was purified

281 from recombinant E. coli. MurG protein (1 or $10 \mu \mathrm{g}$ ) was mixed with lipid I and UDP.

282 GlcNAc, UDP-Gal or UDP-GaINAc (at 0.5 or $5 \mathrm{mM}$ ). The reaction was allowed to

283 proceed for 60 minutes, quenched, and the amount of lipid II produced quantified by

284 TLC. We found that upon addition of $10 \mu \mathrm{g}$ of MurG ( 8 $\mu \mathrm{M})$ and $5 \mathrm{mM}$ UDP-Gal, MurG

285 was able to successfully utilize UDP-Gal as a substrate for the generation of lipid II, with

286 a yield approximately 50\% that of using the correct ligand UDP-GIcNAc (Fig. 4C and

287 Fig. S3). Importantly, galactose and glucose differ by the orientation of the hydroxyl

288 group at the C4 carbon used in the crosslinking of sugars into mature peptidoglycan

289 (Fig. 1A). Formation of lipid II with UDP-Gal (or UDP-GalNAc) in place of the preferred

290 substrate UDP-GIcNAc would thus produce a "toxic" lipid II and could terminate proper

291 peptidoglycan crosslinking. This result indicates that in addition to inhibition of MurG

292 activity, the toxicity could also be due to formation of "toxic" lipid II generated utilizing

293 UDP-Gal as a substrate, and its "poisoning effect" on peptidoglycan polymerization

294 even though the rate of generating "toxic" lipid II could be much slower than that of the

295 native lipid II.

UDP-Gal reduces lipid II production by $S$. aureus MurG in vitro.

298 We wanted to test if UDP-Gal can similarly interfere with the activity of MurG

299 from another bacterium. To do so, MurG of Staphylococcus aureus (MurGsa) was

300 expressed and purified from a recombinant $E$. coli strain ${ }^{34}$. Lipid I was purified using an

301 established biochemical method ${ }^{37}$ and incubated with purified MurGsA and UDP.

302 GlcNAc. The reaction was allowed to proceed, quenched, and the amount of 
synthesized lipid II measured. When UDP-Gal was also added in the reaction as an antagonist (in a ratio of 50:1 to UDP-GIcNAC), MurGsA could convert only $\sim 50 \%$ of lipid I to lipid II compared to no UDP-Gal addition (0.16 $\mu \mathrm{g}$ MurGsA, Fig. 4D). The yield increased to $\sim 70 \%$ if more purified MurGsA protein was added (1.2 $\mu$ g MurGsA, Fig. 4D). Intracellular concentrations of UDP-GIcNAc are estimated at approximately $100 \mu \mathrm{M}$ during the exponential growth ${ }^{38}$, indicating that the concentration of UDP-Gal would have to reach approximately $5 \mathrm{mM}$ (50 fold in excess) to have an $50 \%$ inhibitory effect on MurG, a high concentration, but possible in cells exhibiting toxicity (in which a strong buildup of UDP-Gal is expected). These findings indicate that UDP-Gal can directly

312 interfere the activity of MurGsA at elevated concentrations.

Galactose increases sensitivity of the wild type S. aureus to antibiotics targeting cell wall.

Although accumulation of UDP-Gal during galactose metabolism can occur in the galE mutant, this nucleotide sugar does not accumulate in, nor does it impact, in any noticeable fashion, the WT cells of $B$. subtilis. Galactose metabolism also does not show any toxicity in S. aureus HG003, a model pathogenic strain for clinical infections (data not shown). Interestingly however, when HG003 was grown in the presence of both $0.5 \%(\mathrm{w} / \mathrm{v})$ galactose and the cell wall targeting antibiotic vancomycin (at a subMIC concentration of $0.2 \mu \mathrm{g} / \mathrm{mL}$ ), growth was completely inhibited (Gal, Fig. 4E). Inhibition was not observed when galactose was replaced with the same amount of arabinose (Arab, Fig. 4E). In the absence of either sugar, mildly slower than normal

325 growth was recorded due to the presence of sub-MIC vancomycin (WT, Fig. 4E). One

326 possible explanation for the above synthetic lethality is that upon cell wall stress due to

327 sub-MIC vancomycin treatment, the transient accumulation of UDP-Gal from galactose

328 metabolism started to trigger toxicity even in WT cells, due to synergistic inhibitory

329 actions on cell wall biosynthesis.

To see if our findings extend to Gram-negative species as well, a galE deletion 
334 grown in LB with galactose $(0.5 \%$, w/v), an intermediate toxicity phenotype was

335 observed, judged by a modest decline in cell optical density (red line, Fig. 3E). We were

336 interested in testing if overexpression of the native murG gene in $V$. cholerae could give

337 a similar toxicity rescue seen in B. subtilis. Indeed, the result shown in Fig. 3E suggests

338 that in $V$. cholerae, overexpression of the native murG gene exerted a toxicity rescue

339 phenotype, similar to what we observed in B. subtilis. This result indicates that the

340 toxicity-associated bacterial galactosemia could have a common mechanism in distantly

341 related bacteria.

A suppressor screen on reduced toxicity identifies additional potential targets.

To characterize the additional targets or pathways negatively affected in cells

346 copy of galK and galT genes was introduced into the $B$. subtilis $\Delta$ galE mutant to avoid

347 suppressor mutations in galK or galT that occurred at a very high frequency in previous

348 studies ${ }^{7,39,40}$. Cells were grown on LB agar supplemented with $1 \%(\mathrm{w} / \mathrm{v})$ galactose.

349 Suppressor colonies growing on the plates implied reduced toxicity. They were picked

350 and further verified. A total of 12 suppressor mutants that showed various degrees of

351 toxicity rescue when growing in excess galactose were subjected to whole genome

352 sequencing to characterize the suppressor mutations (Table 1).

We identified a missense mutation in $g t a B(\mathrm{R} 118 \mathrm{P}$, Table 1$)$. The $\mathrm{R} 118$ residue

355 is located next to a highly conserved region $\left({ }^{108} \mathrm{KGLGHAVWCA}^{117}\right)$, which maps to a

356 substrate binding helix essential for catalytic activity in the homologous protein of GtaB

357 in E. coli (sharing $45 \%$ identity in amino acid sequence) ${ }^{41}$. This may further support our

358 finding of the necessity of GtaB activity for toxic UDP-Gal accumulation (Fig. 2B).

359 Suppressor mutations were also found within genes or in regulatory regions of the 360 genes for known sugar or small molecule transport systems: araE, yvfH, ptsG, oppC,

$361 \quad o p p D, \operatorname{tag} H$, and tuaA. Among them, araE encodes an arabinose permease. It is

362 proposed that $B$. subtilis does not possess a dedicated galactose permease and 363 galactose uptake relies on the arabinose permease ${ }^{42}$. Likewise, the hit in araE may

364 serve as another validation of the screen. $y v f H$ was characterized as a L-lactate 
365 permease in our previous study ${ }^{43}$. ptsG encodes the PTS system glucose-specific

366 ElICBA component ${ }^{44}$. oppC and oppD encode the components of an oligopeptide

367 permease in $B$. subtilis ${ }^{45}$. A frame shift mutation was also found in the glcR gene, which

368 very likely caused loss of function of GlcR. GlcR is a transcription repressor for carbon

369 catabolite control in B. subtilis. A deletion mutation in glcR caused derepression of

370 certain carbon catabolite control-repressed genes ${ }^{46}$. Suppressor mutations were also

371 found within a number of cell envelope related genes such as those responsible for wall

372 teichoic acid production $(\operatorname{tagH} \text { and tuaA })^{47}$, as well as those related to cell wall stress

373 response $(\text { cypX and } y r h J)^{48}$. In many of the above cases, how the suppressor mutations

374 impact the activity of the encoded proteins and how their altered activities allow reduced

375 toxicity is unclear and will require further investigation.

\section{Discussion}

378 Bacterial galactosemia was discovered five decades ago, but the mechanism

379 causing toxicity was unclear. Here, we provide evidence for the molecular mechanism

380 of toxicity. We found that metabolism of exogenous galactose in the $B$. subtilis $\Delta g a l E$

381 mutant causes inhibition of cell wall biosynthesis, as determined by MreB filament

382 presence and movement. We show that this toxicity is not caused by inhibition of the

383 essential upstream biosynthetic pathway producing UDP-GIcNAc, instead, accumulation

384 of UDP-Gal can directly interfere with the normal function of MurG. This interference

385 includes not only inhibition of MurG, observed in vivo by imaging of protein diffusion, but

386 possibly the ability of UDP-Gal to be utilized as an unintended substrate by MurG in the

387 generation of a "toxic" lipid II unable to be polymerized into mature peptidoglycan (Fig.

388 4F). We find this mechanism may be shared in $V$. cholerae where overexpression

389 similarly produces a partial rescue, as well as in $S$. aureus, where we present evidence

390 that UDP-Gal is able to directly inhibit MurG activity in vitro.

Previous work has shown that while the production of lipid II is essential, MurG

393 can bind a number of substrates other than UDP-GIcNAc, a property that has been

394 used as the basis of screen for inhibitory compounds of MurG 49,50 . This adds to the

395 possibility of nucleotide sugars such as UDP-Gal as inhibitors or as unintended 
substrates, producing toxic lipid II. The fact that MurG carries out the last cytoplasmic reaction in the peptidoglycan synthesis pathway may add additional lethality if this target is interfered with. To our disappointment, we were unable to identify suppressor mutations in murG in our suppressor screen. Likewise, a targeted approach by using

400 site-directed mutagenesis on selected substrate-binding and catalytic residues in murG

401 aiming to identify such suppressor mutations was also performed but failed to yield 402 positive results (Habib, unpublished). This may be due to the highly essential nature of 403 MurG, making a mutation which further increases specificity for UDP-GlcNAc while still 404 maintaining enzymatic function, impossible.

Given 1) the near ubiquity of peptidoglycan biosynthesis in bacteria, 2)

407 interference of S. aureus MurG-mediated lipid II biosynthesis by UDP-Gal in vitro, and

4083 ) the similar toxicity rescue seen in V. cholerae, it is likely that this mechanism of 409 toxicity is conserved in various bacterial species. It is also possible that this inhibition is 410 not unique to MurG, but to other essential proteins involved in the utilization of UDP411 GlcNAc that may have active site chemistry similar to MurG ${ }^{49}$. Galactosemia was 412 discovered in human century ago ${ }^{51}$. Apparently human cells do not have peptidoglycan 413 and MurG. However, they possess glycocalyx on the cell surface, which functions in 414 cell-cell recognition, communication and adhesion, as well as other functionally 415 important glycoproteins. The synthesis of glycocalyx depends on glycosyltransferases 416 that are functional analogs to bacterial glycosyltransferases such as MurG ${ }^{52}$. It will be 417 interesting to know if the activity of any of those essential glycosyltransferases is altered 418 in the galactosemia patients, though investigation like this is outside the scope of this 419 study.

Finally, in WT B. subtilis, galactose metabolism does not cause toxicity. It is 422 conceivable that when WT cells metabolize galactose, accumulation of UDP-Gal 423 seldomly reaches the threshold that triggers toxicity. As discussed earlier, UDP-Gal 424 production is necessary for several important cellular processes involving various 425 polysaccharide syntheses. Thus, there must be a homeostatic regulation for UPD-Gal production in the bacterium. Many of these nucleotide sugars and phosphorylated 
427 monosaccharides, if accumulated above certain levels, could create an adverse impact 428 on cell physiology or toxicity. Understanding the homeostatic regulation of those sugar 429 intermediates and impact under mis-regulation may reveal new cellular targets and 430 provide new ideas of antibacterial solutions. The galE mutant used in this study can be 431 considered an unusual case, in which there is misregulation of specific sugar

432 metabolites. Under this misregulation, we discovered that the cytoplasmic stages of cell 433 wall biosynthesis can indeed be the targets of sugars native to the cells. Using such an 434 artificial system conveniently allows us to understand the key points of regulation as 435 well as potential novel antibacterial ideas. Finally, although galactose metabolism does

436 not cause any toxicity in WT cells, the increase in sensitivity to cell-wall targeting 437 antibiotics exhibited in the WT S. aureus upon simultaneous galactose metabolism 438 demonstrates possible clinical relevance of galactose metabolism-induced cellular 439 stress or toxicity.

Materials and Methods

442 Strains, medias, and reagents. B. subtilis and E. coli strains were routinely cultured in 443 lysogeny broth (LB). All strains used in this study are listed in Table S1. Insertional 444 deletion strains, if not specified in the methods, were purchased from the Bacillus 445 Genome Stock Center (BGSC, Columbus, OH). Insertional deletion mutations were 446 then introduced into the $B$. subtilis NCIB3610 background by transformation. MSgg 447 media for biofilm formation was prepared according to the published protocol ${ }^{53}$. To 448 induce the toxicity, cells were grown in LB (1\% tryptone, $0.5 \%$ yeast extract, and $1 \%$ $449 \mathrm{NaCl}$ ) with the addition of $0.5 \%(\mathrm{w} / \mathrm{v})$ galactose and $0.01 \%(\mathrm{w} / \mathrm{v})$ arabinose. Addition of 450 arabinose is to induce the araE-encoded arabinose permease for galactose uptake ${ }^{42}$. If 451 required, antibiotics were added at the following concentrations for $B$. subtilis: $10 \mu \mathrm{g} \mathrm{ml}^{-1}$ 452 kanamycin, $50 \mu \mathrm{g} \mathrm{ml}^{-1}$ spectinomycin, and $5 \mu \mathrm{g} \mathrm{ml}^{-1}$ tetracycline. For $E$. coli strains, 453 when required, antibiotics were used at: $100 \mu \mathrm{g} \mathrm{ml}^{-1}$ ampicillin and at $50 \mu \mathrm{g} \mathrm{ml}^{-1}$ 454 kanamycin. Chemicals, including galactose (CAS No. 59-23-4), arabinose (CAS No. 455 5328-37-0), UDP-NAc-Glucosamine (CAS No. 91183-98-1), and UDP-Galactose (CAS 456 No. 137868-52-1) were purchased from Sigma-Aldrich. Restriction enzymes were 457 purchased from New England Bio-labs (NEB, MA, USA). Oligonucleotides (sequences 
458 provided in Table S2) were purchased from Integrated DNA Technologies (IDT, IA,

459 USA). DNA sequencing was performed at Genewiz (NJ, USA).

Strain construction was described in Supplementary Materials.

Biofilm development. For pellicle biofilm development, the $\Delta g t a B$ cells were inoculated from colonies on an overnight LB agar plate into $3 \mathrm{~mL}$ of LB broth and grown with shaking at $37^{\circ} \mathrm{C}$ to log phase. Cells were then subcultured 1:1000 into $7 \mathrm{~mL}$ of MSgg in a 6-well polyvinyl plate (VWR, PA, USA). Plates were incubated in static conditions at $30^{\circ} \mathrm{C}$ for 3 days and were then imaged using a Leica MZ10F macroscope and Leica DMC2900 camera. For colony biofilm development, $\Delta$ gtaB cells were similarly prepared and $3 \mathrm{ul}$ of the log phase cells was spotted onto the MSgg media solidified with $1.5 \%$ agar (w/v). Plates were incubated at $30^{\circ} \mathrm{C}$ for 3 days and were then imaged using a Nikon Coolpix camera.

Phase contrast microscopy. Phase contrast and fluorescent imaging for live/dead staining was performed on a Leica DM5000B light microscope. Cells were grown to mid-exponential phase and collected by centrifugation for 1 minute at 16,000 $\mathrm{g}$, resuspended in phosphate buffered solution (PBS), and concentrated 10-fold. $2 \mu \mathrm{L}$ of the resuspended culture was spotted on a glass slide and covered with poly-L-lysine coated coverslip. Resulting slides were viewed at 100x magnification and images were captured with Leica DFC300G camera. To perform live / dead staining, cells were collected, concentrated as above, and treated with live/dead staining dyes (Invitrogen, Carlsbad, CA) according to manufacturer's protocol with resulting samples imaged under GFP (for live cells) and Texas Red (for dead cells) fluorescent filters. The ratio of live/dead cells was manually counted for about a total of 300 cells.

Scanning Electron Microscopy. B. subtilis $\Delta$ galE cells were grown overnight in LB to stationary phase and subcultered 1:100 in LB supplemented with galactose and were 487 grown 3 hours shaking at $37^{\circ} \mathrm{C}$. Cells were collected via centrifugation at 5,000 g and washed twice with PBS. Cells were allowed to adhere to a glass coverslip treated with 
poly-L-lysine for 1 hour. Adhered cells were fixed in a solution containing $2.5 \%$ glutaraldehyde and $0.1 \mathrm{M}$ sodium cacodylate at $\mathrm{pH} 7.2$ for 1 hour and then rinsed for 10 minutes in 0.1 sodium cacodylate buffer $\mathrm{pH} 7.2$ three times. Cells were infiltrated with $1 \%$ osmium tetroxide in $0.1 \mathrm{M}$ sodium cacodylate $\mathrm{pH} 7.2$ for 1 hour and then washed again three times with 0.1 sodium cacodylate buffer $\mathrm{pH} 7.2$ for 10 minutes each wash. Cells were then dehydrated in ethanol at 30\%,70\%, 85\%, and 95\%, for 10 minutes, and finally in $100 \%$ ethanol for three times at 10 minutes each wash. Samples were then dried in a critical point dryer utilizing carbon dioxide, mounted to specimen mounts, using double-sided carbon adhesive tape, and sputter-coated with $5 \mathrm{~nm}$ platinum. Samples were then examined using a high-resolution field emission SEM Hitachi S4800 under vacuum using an acceleration voltage of $>2.0 \mathrm{kV}$.

Single-cell growth rate time lapse experiments. $2-\mu \mathrm{L}$ culture was spotted on No. 1.5 glass-bottomed dishes (MatTek, MA) under 3\% agarose pads made with casein hydrolysate $(\mathrm{CH})$ medium. The phase-contrast images were collected on a Nikon Ti microscope equipped with a Hamamatsu ORCA Flash4 CMOS camera with a Nikon Plan Apo $\lambda$ 100×/1.4NA objective. Images were acquired from 50 fields of view every 2 min for a total of 2-3 hour using NI Element. The phase-contrast time-lapse movies were analyzed using a custom-built package in MATLAB.

Imaging of MreB-mNeonGreen by TIRF microscopy. Cells were placed under a $\mathrm{CH}$ agarose pad with galactose $(0.5 \%, \mathrm{w} / \mathrm{v})$ on a glass coverslip for imaging. Images were collected on a Nikon Ti microscope equipped with a Hamamatsu ORCA Flash4 CMOS camera with a Nikon 100X NA 1.45 objective. Exposure time is 300ms and the time

513 interval 1 second. A 488nm laser line is used for the imaging of mNeonGreen. The 514 method used to analyze the density of MreB filaments has been published previously ${ }^{28}$.

515 First the phase images were segmented, and the fluorescence time-lapses were 516 analyzed based on the segmentation mask of the phase image. Next for each cell the 517 kymographs were generated for each row of pixels along the midline of the cell, and the 518 time-lapse movie was converted into a single 2D image. Then the MreB filament were 519 registered in the $2 \mathrm{D}$ image. The MreB filament density is normalized to the density in 
520 WT cells (YS09). All of the image analyses were performed using custom MATLAB 521 code.

523 Imaging of HaloTag-MurG by TIRF microscopy. HaloTag-MurG cells were incubated 524 with 50 pM of HaloTag-JF549 ligand for 30 minutes and then washed twice to get rid of 525 free dyes. Images were collected on a Nikon Ti microscope equipped with a 526 Hamamatsu ORCA Flash4 CMOS camera with a Nikon 100X NA 1.45 objective.

527 Exposure time is $300 \mathrm{~ms}$ and the time interval 1 second. A $561 \mathrm{~nm}$ laser line is used for 528 the imaging of JF549-HALO dye.

530 Bulk growth analysis. For bulk culture growth measurements by cell optical density

$531\left(\mathrm{OD}_{600}\right)$, cells were grown overnight in LB plus applicable antibiotics to stationary phase 532 with shaking at $200 \mathrm{rpm}$ at $37^{\circ} \mathrm{C}$. Cells were subcultured 1:100 in respective media and 533 grown to mid exponential phase $\left(\mathrm{OD}_{600}=0.4-0.5\right)$. Cells were subcultured 1:100 in 96534 well tissue culture plates with $170 \mu \mathrm{L}$ final volume of media in each well. Plates were 535 read by BioTek plate reader with shaking at $200 \mathrm{rpm}$ and incubating at $37^{\circ} \mathrm{C}$ with $\mathrm{OD}_{600}$ 536 readings taken even 15 minutes. Experiments were performed at least three times and representative data set were shown.

To determine the effect of galactose metabolism on growth of Staphylococcus

539 aureus, cells were grown shaking at $200 \mathrm{rpm}$ at $37^{\circ} \mathrm{C}$ overnight. Cells were subcultured $540 \quad$ 1:200 in LB diluted 1:4 with sterile water. Vancomycin was added to all samples at 0.2 $541 \mu \mathrm{g} / \mathrm{mL}$, and galactose or arabinose was added at $0.5 \%(\mathrm{w} / \mathrm{v})$, with sterile water used as 542 a control. Samples were grown in a 24-well tissue culture plate with $2 \mathrm{~mL}$ final volume in 543 each well and read as above.

545 Peptidoglycan precursor measurement. Measurement of peptidoglycan precursor 546 was performed using published methods with minor modifications ${ }^{35}$. Briefly, $B$. subtilis 547 strain $\mathrm{CH} 62$ was grown overnight in LB with tetracycline to stationary phase and 548 subculture 1:100 into both LB and LB plus 0.5\% (w/v) galactose. Vancomycin was used 549 as a control at $10 \mu \mathrm{g} \mathrm{mL}^{-1}$ added at $\mathrm{OD}_{600}=0.4$ and were treated for 30 minutes before 550 collection. $10 \mathrm{~mL}$ of cells from both conditions were collected every 30 minutes and 
551 spun down for 10 minutes at $16,000 \mathrm{~g}$. Total lipid content of the cells was extracted via

552 two-phase system and concentrated in vacuo. The resulting extract was mixed with

553 biotinylated lysine (BDL) probe and purified PBP4 protein and allowed to react for an

554 hour at $25^{\circ} \mathrm{C}$ before being quenched. Reaction mixture was loaded on to a $15 \%$ SDS-

555 PAGE and transferred to Immuno-Blot PVDF membrane. BDL was detected utilizing HRP-Conjugated Streptavidin antibody and imaged on BioRad ChemiDoc MP. The resulting gel was quantified for pixel density using ImageJ. Experiment was performed

558 three times and a representative image is presented.

MurG in vitro assay. MurG activity using different UDP-activated substrates was tested as previously described with minor modifications ${ }^{34}$. Briefly, MurG activity assays were performed in a final volume of $30 \mu \mathrm{L}$ containing $3 \mathrm{nmol}$ of purified lipid I, 5.8 to $50 \mathrm{mM}$ of either UDP-Gal or UDP-GalNAc in $60 \mathrm{mM}$ Tris-HCl, $5 \mathrm{mM} \mathrm{MgCl2,} \mathrm{pH} 7.5$, and 0.5\%

564 Triton X-100 in the presence of 1 or $10 \mu \mathrm{g}(\sim 0.8$ or $8 \mu \mathrm{M})$ of purified MurG $\mathrm{Bsub}_{-}$

565 His 6 proteins. The reaction mixture was incubated for $60 \mathrm{~min}$ at $30{ }^{\circ} \mathrm{C}$. Lipid intermediates were extracted with an equal volume of n-butanol/pyridine acetate, $\mathrm{pH} 4.2$ $(2: 1 ; \mathrm{v} / \mathrm{v})$ and analyzed by thin layer chromatography (TLC) using chloroform/methanol/water/ammonia (88:48:10:1, v/v/v/v) as the solvent ${ }^{34}$. The quantitative analysis of lipid II extracted to the butanol phase was carried out using ImageJ ${ }^{54}$. The inhibitory effect of UDP-Gal (molar ratio of 50:1 with respect to UDPGlcNAc) on MurGsA activity was tested in a final volume of $30 \mu \mathrm{L}$ containing $1 \mathrm{nmol}$ of purified lipid I, $10 \mathrm{nmol}$ of UDP-GIcNAc, $0.1 \mathrm{nmol}$ [14C]UDP-GIcNAc (1.23 kBq) in 60 $\mathrm{mM}$ Tris- $\mathrm{HCl}, 5 \mathrm{mM} \mathrm{MgCl}$, $\mathrm{pH} 7.5$, and $0.5 \%$ Triton $\mathrm{X}-100$ in the presence of 0.16 or

$5741.2 \mu \mathrm{g}$ of purified MurG-His 6 protein. The reaction mixture was incubated for $30 \mathrm{~min}$ at $30{ }^{\circ} \mathrm{C}$. Lipid intermediates were extracted and analyzed by thin layer chromatography (TLC) as described above. Radiolabeled lipids were visualized by phosphorimaging using a Storm imaging system and quantification was performed using the software ImageQuant TL (GE Healthcare), 


\section{Acknowledgments}

583 We would like to thank Luke Lavis for JF dyes, and Dr. Kim Lewis (Northeastern

584 University) for the gift of teixobactin. This work was supported by a grant from the

585 National Science Foundation (MCB1651732) to YC. This work was also funded by

586 National Institutes of Health Grants DP2AI117923-01 to EG, as well as support from the

587 Volkswagen Foundation. Funding was also provided by the German Research

588 Foundation (Project-ID 398967434 - TRR261 to T.S. and A.M) and an NIH grant

589 (Al120489) to JZ.

590

\section{Author contributions}

592 C.H., Y.S., E.G., and Y.C. conceived the project; C.H. and Y.S. cloned the strains; C.H.

593 A.M., T.S., M.L., and Y.S. planned and performed the experiments; C.H., A.M., Y.S.,

594 and Y.C. analyzed the data; Y.S. wrote the code for the single cell analyses; C.H., Y.S.,

595 and Y.C. wrote the manuscript, with contributions and editing from all authors.

\section{Competing interests}

598 The authors declare no competing interests.

\section{References}

6021 Frey, P. The Leloir pathway: A mechanistic imperative for three enzymes to change the stereochemical configuration of a single carbon in galactose. FASEB journal : official publication of the Federation of American Societies for Experimental Biology 10, 461-470, doi:10.1096/fasebj.10.4.8647345 (1996).

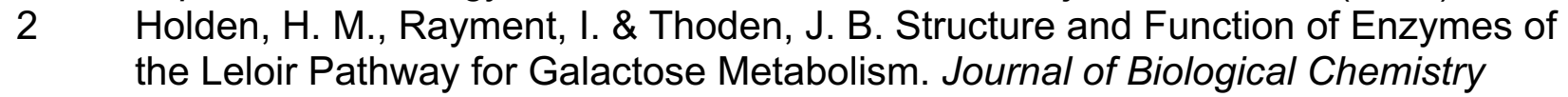
278, 43885-43888, doi:10.1074/jbc.R300025200 (2003).

3 Fukasawa, T. \& Nikaido, H. Galactose-sensitive mutants of Salmonella. Nature 184, 1168-1169 (1959).

4 Yarmolinsky, M. B., Wiesmeyer, H., Kalckar, H. M. \& Jordan, E. Hereditary defects in galactose metabolism in Escherichia coli mutants, II. Galactoseinduced sensitivity. . Proceedings of the National Academy of Sciences of the United States of America 45, 1786-1791, doi:10.1073/pnas.45.12.1786 (1959).

5 Barkan, D., Stallings, C. L. \& Glickman, M. S. An improved counterselectable marker system for mycobacterial recombination using galK and 2-deoxygalactose. Gene 470, 31-36 (2011). 
6186 Krispin, O. \& Allmansberger, R. The Bacillus subtilis galE gene is essential in the 619 presence of glucose and galactose. Journal of bacteriology 180, 2265-2270 (1998).

7 Chai, $Y$ Chai, Y., Beauregard, P. B., Vlamakis, H., Losick, R. \& Kolter, R. Galactose Metabolism Plays a Crucial Role in Biofilm Formation by Bacillus subtilis. mBio 3, e00184-00112, doi:10.1128/mBio.00184-12 (2012).

8 Beauregard, P. B., Chai, Y., Vlamakis, H., Losick, R. \& Kolter, R. Bacillus subtilis biofilm induction by plant polysaccharides. Proceedings of the National Academy of Sciences 110, E1621-E1630 (2013).

9 Labavitch, J. M., Freeman, L. E. \& Albersheim, P. Structure of plant cell walls. Purification and characterization of a beta-1,4-galactanase which degrades a structural component of the primary cell walls of dicots. Journal of Biological Chemistry 251, 5904-5910 (1976).

10 Watzlawick, H., Morabbi Heravi, K. \& Altenbuchner, J. Role of the ganSPQAB Operon in Degradation of Galactan by Bacillus subtilis. Journal of Bacteriology 198, 2887-2896, doi:10.1128/jb.00468-16 (2016).

11 Habib, C. et al. Characterization of the regulation of a plant polysaccharide utilization operon and its role in biofilm formation in Bacillus subtilis. PLOS ONE 12, e0179761, doi:10.1371/journal.pone.0179761 (2017).

12 Chen, Y. et al. A Bacillus subtilis sensor kinase involved in triggering biofilm formation on the roots of tomato plants. Mol. Microbiol. 85, 418-430, doi:10.1111/j.1365-2958.2012.08109.x (2012). natural environments depends on conserved genes mediating biofilm formation. Environmental Microbiology 15, 848-864, doi:10.1111/j.1462-2920.2012.02860.x (2013).

14 Mengin-Lecreulx, D., Texier, L., Rousseau, M. \& van Heijenoort, J. The murG gene of Escherichia coli codes for the UDP-N-acetylglucosamine: Nacetylmuramyl-(pentapeptide) pyrophosphoryl-undecaprenol Nacetylglucosamine transferase involved in the membrane steps of peptidoglycan synthesis. Journal of bacteriology 173, 4625-4636, doi:10.1128/jb.173.15.46254636.1991 (1991).

15 van Heijenoort, J. Lipid Intermediates in the Biosynthesis of Bacterial Peptidoglycan. Microbiology and Molecular Biology Reviews 71, 620-635, doi:10.1128/mmbr.00016-07 (2007).

16 Meeske, A. J. et al. MurJ and a novel lipid II flippase are required for cell wall biogenesis in Bacillus subtilis. Proceedings of the National Academy of Sciences of the United States of America 112, 6437-6442, doi:10.1073/pnas.1504967112 (2015).

17 Ruiz, N. Bioinformatics identification of MurJ (MviN) as the peptidoglycan lipid II flippase in Escherichia coli. Proceedings of the National Academy of Sciences 105, 15553-15557, doi:10.1073/pnas.0808352105 (2008).

661 Sham, L.-T. et al. MurJ is the flippase of lipid
biogenesis. Science 345, 220-222 (2014). 
66219 Schirner, K. et al. Lipid-linked cell wall precursors regulate membrane

663

664

665

666

667

668

669

670

671

672

673

674

675

676

677

678

679

680

681

682

683

684

685

686

687

688

689

690

691

692

693

694

695

696

697

698

699

700

701

702

703

704

705

706

707

association of bacterial actin MreB. Nature Chemical Biology 11, 38-45,

doi:10.1038/nchembio.1689 (2014).

20 Shi, H., Bratton, B. P., Gitai, Z. \& Huang, K. C. How to Build a Bacterial Cell: MreB as the Foreman of E. coli Construction. Cell 172, 1294-1305, doi:10.1016/j.cell.2018.02.050 (2018).

21 Garner, E. C. et al. Coupled, Circumferential Motions of the Cell Wall Synthesis Machinery and MreB Filaments in B. subtilis. Science 333, 222-225, doi:10.1126/science.1203285 (2011).

22 Berbis, M. A., Jose Maria, S.-P., Canada, F. J. \& Jesus, J.-B. Structure and Function of Prokaryotic UDP-Glucose Pyrophosphorylase, A Drug Target Candidate. Current Medicinal Chemistry 22, 1687-1697, doi:http://dx.doi.org/10.2174/0929867322666150114151248 (2015).

23 Chien, A.-C., Hill, Norbert S. \& Levin, Petra A. Cell Size Control in Bacteria. Current Biology 22, R340-R349, doi:https://doi.org/10.1016/i.cub.2012.02.032 (2012).

24 Lee, S. J. et al. Cellular stress created by intermediary metabolite imbalances. Proceedings of the National Academy of Sciences of the United States of America 106, 19515-19520, doi:10.1073/pnas.0910586106 (2009).

25 Varón, D., Boylan, S. A., Okamoto, K. \& Price, C. W. Bacillus subtilis gtaB encodes UDP-glucose pyrophosphorylase and is controlled by stationary-phase transcription factor sigma B. Journal of Bacteriology 175, 3964-3971, doi:10.1128/jb.175.13.3964-3971.1993 (1993).

26 Jones, L., J. F. , Carballido, R. \& Errington, J. Control of Cell Shape in Bacteria: Helical, actin-like filaments in Bacillus subtilis. Cell 104, 913-922, doi:10.1016/S0092-8674(01)00287-2 (2001).

27 Hussain, S. et al. MreB filaments align along greatest principal membrane curvature to orient cell wall synthesis. eLife 7, 1239, doi:10.7554/eLife.32471 (2018).

28 Dion, M. F. et al. Bacillus subtilis cell diameter is determined by the opposing actions of two distinct cell wall synthetic systems. Nature Microbiology 4, 12941305, doi:10.1038/s41564-019-0439-0 (2019).

29 Patel, V., Wu, Q., Chandrangsu, P. \& Helmann, J. D. A metabolic checkpoint protein GImR is important for diverting carbon into peptidoglycan biosynthesis in Bacillus subtilis. PLOS Genetics 14, e1007689, doi:10.1371/journal.pgen.1007689 (2018).

30 Foulquier, E. \& Galinier, A. YvcK, a protein required for cell wall integrity and optimal carbon source utilization, binds uridine diphosphate-sugars. Scientific Reports 7, 4139, doi:10.1038/s41598-017-04064-2 (2017).

31 Brown, E. D., Vivas, E. I., Walsh, C. T. \& Kolter, R. MurA (MurZ), the enzyme that catalyzes the first committed step in peptidoglycan biosynthesis, is essential in Escherichia coli. Journal of Bacteriology 177, 4194-4197, doi:10.1128/jb.177.14.4194-4197.1995 (1995).

32 Chung, B. C. et al. Crystal structure of MraY, an essential membrane enzyme for bacterial cell wall synthesis. Science (New York, N.Y.) 341, 1012-1016, doi:10.1126/science.1236501 (2013). 
33 Bouhss, A., Trunkfield, A. E., Bugg, T. D. H. \& Mengin-Lecreulx, D. The biosynthesis of peptidoglycan lipid-linked intermediates. FEMS Microbiology Reviews 32, 208-233, doi:10.1111/j.1574-6976.2007.00089.x (2008). Mann, P. A. et al. Murgocil is a Highly Bioactive Staphylococcal-Specific Inhibitor of the Peptidoglycan Glycosyltransferase Enzyme MurG. ACS Chemical Biology 8, 2442-2451, doi:10.1021/cb400487f (2013).

35 Qiao, Y. et al. Detection of Lipid-Linked Peptidoglycan Precursors by Exploiting an Unexpected Transpeptidase Reaction. Journal of the American Chemical Society 136, 14678-14681, doi:10.1021/ja508147s (2014).

36 Ling, L. L. et al. A new antibiotic kills pathogens without detectable resistance. Nature 517, 455-459, doi:10.1038/nature14098 (2015).

37 Schneider, T. et al. In vitro assembly of a complete, pentaglycine interpeptide bridge containing cell wall precursor (lipid II-Gly5) of Staphylococcus aureus. Molecular microbiology 53, 675-685 (2004).

38 Mengin-Lecreulx, D., Flouret, B. \& van Heijenoort, J. Pool levels of UDP Nacetylglucosamine and UDP $\mathrm{N}$-acetylglucosamine-enolpyruvate in Escherichia coli and correlation with peptidoglycan synthesis. Journal of bacteriology 154, 1284-1290 (1983).

39 Shuster, C. W. \& Rundell, K. Resistance of Salmonella typhimurium mutants to galactose death. Journal of bacteriology 100, 103-109 (1969).

40 Germanier, R. \& Fürer, E. Immunity in Experimental Salmonellosis II. Basis for the Avirulence and Protective Capacity of galE Mutants of Salmonella typhimurium. Infection and Immunity 4, 663-673 (1971).

41 Thoden, J. B. \& Holden, H. M. The molecular architecture of glucose-1phosphate uridylyltransferase. Protein Sci 16, 432-440, doi:10.1110/ps.062626007 (2007).

42 Krispin, O. \& Allmansberger, R. The Bacillus subtilis AraE protein displays a broad substrate specificity for several different sugars. Journal of bacteriology 180, 3250-3252 (1998).

43 Chai, Y., Kolter, R. \& Losick, R. A Widely Conserved Gene Cluster Required for Lactate Utilization in Bacillus subtilis and Its Involvement in Biofilm Formation.

44 Reizer, J. et al. Novel phosphotransferase system genes revealed by genome analysis - the complete complement of PTS proteins encoded within the genome of Bacillus subtilis. Microbiology 145, 3419-3429, doi:doi:10.1099/00221287-14512-3419 (1999).

45 Perego, M., Higgins, C. F., Pearce, S. R., Gallagher, M. P. \& Hoch, J. A. The oligopeptide transport system of Bacillus subtilis plays a role in the initiation of sporulation. Mol. Microbiol. 5, 173-185, doi:10.1111/j.1365-2958.1991.tb01838.x (1991).

46 Stülke, J., Martin-Verstraete, I., Philippe, G. \& G, R. Characterization of glucoserepression-resistant mutants of Bacillus subtilis: Identification of the glcR gene. Archives of microbiology 175, 441-449, doi:10.1007/s002030100288 (2001).

47 Brown, S., Santa Maria Jr., J. P. \& Walker, S. Wall Teichoic Acids of GramPositive Bacteria. Annu. Rev. Microbiol. 67, 313-336, doi:10.1146/annurev-micro092412-155620 (2013). 
48 Mascher, T., Margulis, N. G., Wang, T., Ye, R. W. \& Helmann, J. D. Cell wall stress responses in Bacillus subtilis: the regulatory network of the bacitracin stimulon. Molecular Microbiology 50, 1591-1604, doi:10.1046/j.13652958.2003.03786.x (2003).

$49 \mathrm{Hu}, \mathrm{Y}$. et al. Crystal structure of the MurG:UDP-GlcNAc complex reveals common structural principles of a superfamily of glycosyltransferases. Proceedings of the National Academy of Sciences of the United States of America 100, 845-849, doi:10.1073/pnas.0235749100 (2003).

$50 \mathrm{Hu}, \mathrm{Y}$. et al. Identification of Selective Inhibitors for the Glycosyltransferase MurG via High-Throughput Screening. Chemistry \& Biology 11, 703-711, doi:https://doi.org/10.1016/j.chembiol.2004.02.024 (2004).

51 Leslie, N. D. Insights into the pathogenesis of galactosemia. . Annual Review of Nutrition 23, 59-80, doi:10.1146/annurev.nutr.23.011702.073135 (2003).

52 Spiro, R. G. Protein glycosylation: nature, distribution, enzymatic formation, and disease implications of glycopeptide bonds. Glycobiology 12, 43R-56R, doi:10.1093/glycob/12.4.43R (2002).

53 Branda, S. S., Gonzalez-Pastor, J. E., Ben-Yehuda, S., Losick, R. \& Kolter, R. Fruiting body formation by Bacillus subtilis. Proc. Natl. Acad. Sci. USA 98, 11621-11626, doi:10.1073/pnas.191384198 (2001).

54 Schneider, C. A., Rasband, W. S. \& Eliceiri, K. W. NIH Image to ImageJ: 25 years of image analysis. Nature Methods 9, 671-675, doi:10.1038/nmeth.2089 (2012).

\section{Figure Legends}

\section{Figure 1. Incomplete galactose metabolism causes cell shape deformation and}

cell lysis in B. subtilis. (A) An overview of the Leloir pathway in B. subtilis. Galactose metabolism is carried out by three enzymes, galactokinase (GalK), which first catalyzes a-D-galactose to galactose-1-phosphate, followed by galactose-1-phosphate uridylyltransferase (GalT), which transfers a uridine-diphosphate group from UDP-glucose to galactose-1-phosphate, forming UDP-galactose and glucose-1-phosphate. Finally, UDP glucose 4-epimerase (GalE) catalyzes the reversible reaction from UDP-galactose to UDP-glucose. In addition, a UTP-glucose-1-phosphate uridylyl-transferase (GtaB) replenishes the pool of UDP-glucose by catalyzing the reaction from glucose-1phosphate to UDP-glucose. The two sugar epimers, $\alpha$-D-Glucose and $\alpha$-D-galactose, differ only in the orientation of the hydroxyl group at $\mathrm{C} 4$ carbon (indicated by arrows). (B) The $\triangle$ galE mutant of $B$. subtilis $(\mathrm{CH} 62)$ was grown in LB and $\mathrm{LB}+$ galactose $(0.5 \%$, $w / v$ ) to log phase while shaking at $37^{\circ} \mathrm{C}$. Cells were collected, stained for live (green) / 
792 dead (red) fluorescence dyes, and imaged. The percentage of live / dead cells was 793 quantified (shown below panels). Scale bar is $10 \mu \mathrm{m}$. (C) Cells were imaged under

794 phase contrast microscopy to determine the effect of the toxicity on cell morphology.

795 Structural defects (red arrows) including bulges, rounding out of bacilli shape, and cell

796 lysis were observed. Scale bar is $4 \mu \mathrm{m}$. (D) Scanning electron microscopy shows

797 structural defects of cells, which ranged from shape deformities to complete loss of

798 integrity. Scale bar is $1 \mu \mathrm{m}$. (E) After addition of galactose the progression of toxicity

799 was measured over time as a function of maximal cell width of $\sim 100$ cells using

800 microscopy. Increasing maximal width over time indicates more severe structural

801 defects associated with incomplete galactose metabolism. Error bars represent

802 standard deviations.

803

804 Figure 2. (A-B) GtaB is required for toxicity. (A) WT B. subtilis (3610) and a strain 805 deficient in gtaB (YC876) were grown in LB shaking at $37^{\circ} \mathrm{C}$ and $\mathrm{OD}_{600}$ values of the 806 cultures were measured every 15 minutes for a period of 8 hours. Representative 807 biological replicate shown with error bars representing standard deviation. (B) An IPTG 808 inducible copy of $g t a B$ was introduced into the $\triangle g a l E \Delta g t a B$ double mutant and the result 809 strain $(\mathrm{CH} 117)$ was grown in shaking LB with galactose $(0.5 \%, w / v)$ and varying 810 amounts of IPTG for gtaB induction, together with the WT (3610) and the $\triangle$ galE mutant

811 (CH62). As judged by the OD 600 values of the cultures. Representative biological

812 replicate shown with error bars representing standard deviation. (C-F) Toxicity is due

813 to inhibition of cell wall biosynthesis. (C-D) Kymograph of MreB movement in the

$814 \Delta$ galE mutant. The polymerization and movement of MreB filaments is tracked using

815 MreB-mNeonGreen fusions, a motion dependent upon active cell wall synthesis. $\Delta g a l E$

816 cells expressing the MreB-mNeonGreen fusion protein were immobilized on a $\mathrm{CH}$ agar

817 pad with galactose $(0.5 \%, \mathrm{w} / \mathrm{v})$ and imaged with time lapse TIRF every 30 minutes. The 818 cellular MreB-mNeonGreen location was recorded (C), analyzed, then plotted (D). The 819 number of MreB-mNeonGreen filaments was found to substantially decrease over time, 820 dissolving completely 60 minutes after galactose exposure. (E-F) Kymograph of MreB 821 movement in the $\Delta$ galE mutant with murG overexpression (1 mM IPTG). (E) MreB

822 filament formation and movement was recorded similarly in the $\triangle$ galE cells 
823 overexpressing murG by growth on agar pad containing galactose $(0.5 \%$, w/v) and 500

$824 \quad \mu \mathrm{M}$ IPTG. (F) The number of MreB filaments was found partially restored by murG

825 overexpression in 60,75 , and 90 min samples.

826

827 Figure 3. (A-B) Toxicity is not likely due to inhibition in synthesis of UDP-GIcNAc.

828 (A) The biosynthetic pathway of UDP-GIcNAc and UDP-MurNAc. GImS, GImM, GImU,

829 MurAA are enzymes that carry out specific reactions from Fru-6-P to UDP-GIcNAc and

830 UDP-MurNAc. GImR is a regulatory protein for GImS and the activity of GImR is

831 negatively regulated by UDP-GIcNAc. (B) The $\Delta$ galE mutant bearing either an IPTG-

832 inducible copy of $g / m S$, glmR, glmM, or $g / m U$ involved in UDP-GlcNAc biosynthesis was

833 grown in LB shaking broth in a plate reader. OD 600 of the cultures was measured

834 periodically. Representative biological replicate shown with error bars representing

835 standard deviation. (C-E) MurG is a possible direct target of inhibition in cells

836 exhibiting toxicity. (C) An IPTG inducible copy of murG was introduced into the $B$.

837 subtilis $\Delta$ galE mutant $(\mathrm{CH} 112)$ and grown in LB shaking at $37^{\circ} \mathrm{C}$ with or without

838 galactose $(0.5 \%, w / v)$ and $0.2 \mathrm{mM}$ IPTG, along with the WT and $\Delta$ galE strains in LB

839 with galactose. OD 600 values were measured every 15 minutes. Representative

840 biological replicate shown with error bars representing standard deviation. (D) The

841 effect of murG overexpression on growth rate and toxicity rescue at the single cell

842 resolution. The $\triangle$ galE murG overexpression strain $(\mathrm{CH} 112)$ was plated on a $\mathrm{CH}$ agar

843 pad containing galactose $(0.5 \%, \mathrm{w} / \mathrm{v})$ and $0,50,500$, and $1000 \mu \mathrm{M}$ of IPTG and imaged

844 every 15 minutes to determine growth rate as a function of doublings per hour. WT cells

845 were used as a control. (E) A similar murG overexpression strain was constructed in the

$846 V$. cholerae $\Delta$ galE mutant using its native murG gene. Cells were grown in LB shaking

847 broth and $\mathrm{OD}_{600}$ values were measured periodically. murG $\mathrm{vc}_{c}$ was overexpressed upon

848 addition of $500 \mu \mathrm{M}$ IPTG. Strains used for the $V$. cholerae assay include WT(C6706),

$849 \Delta$ galE(VCA0774), and $\Delta$ galE-murG ${ }^{\text {OV }}(\mathrm{VC} 2401)$. Error bar represents standard

850 deviation.

851 Figure 4. Toxicity is due to block in synthesis of lipid precursors. (A)

852 Peptidoglycan lipid precursor levels were measured using a published protocol ${ }^{35}$. The

853 galE mutant $(\mathrm{CH} 62)$ was grown without $(-)$ and with $(+)$ galactose $(0.5 \%, w / v)$ in shaking 
854 cultures and samples were collected every 30 minutes. Total cell lipids were extracted, 855 biotinylated, and visualized by western blot. As a control, cells treated with cell wall 856 antibiotic vancomycin were similarly assayed. (B) The above gel was analyzed, and the 857 amount of lipid I / II quantified by calculating the pixel density using Image J. Assays 858 were repeated three times. A representative result is shown in A and pixel density 859 quantification shown in B. (C) To determine if UDP-Gal could be used as a substrate by 860 MurG and incorporated into lipid II, B. subtilis MurG was mixed with lipid I and UDP-Gal, 861 UDP-GalNAc, or UDP-GIcNAc, and the amount of resulting lipid II quantified. Upon 862 addition of $5 \mathrm{mM}$ UDP-Gal, there was a $50 \%$ production of lipid II as compared to the 863 control of UDP-GIcNAc. Additionally, there was an approximately $7 \%$ production of lipid 864 II upon addition of UDP-GalNAc. (D) The lipid II yield was measured by using purified S. aureus MurGsA and allowing it to react with UDP-GIcNAc without and with the addition

866 of UDP-Gal in an in vitro lipid II synthesis assay. Upon addition of UDP-Gal (5 mM) in a 867 ratio of $50: 1$ to UDP-GIcNAc $(100 \mu \mathrm{M}$ ), production of lipid II decreased $50 \%$ (at $0.16 \mu \mathrm{g}$ 868 MurG). The decrease became milder ( 30\%) if 7.5-fold more purified MurGsa was 869 added (at 1.2 $\mu \mathrm{g}$ MurG). Representative biological replicate shown with error bars 870 representing standard deviation. (E) Synergistic lethality of galactose and cell-wall 871 targeting antibiotic vancomycin on WT S. aureus cells. The WT S. aureus HG003 was 872 grown in 1:4 diluted LB with a sub-MIC concentration $(0.2 \mu \mathrm{g} / \mathrm{mL})$ of vancomycin.

873 Without addition of galactose, growth was slower than normal, due to sub-MIC 874 vancomycin (WT). Upon addition of $0.5 \%$ galactose, growth was completely inhibited 875 (Gal). This inhibition was not seen during addition of $0.5 \%$ arabinose (Arab). 1:4 diluted

876 LB broth was used here to minimize catabolite repression of galactose metabolism 877 genes in S. aureus. (F) A cartoon demonstration of how UDP-Gal accumulation inhibits 878 cell wall biosynthesis in bacteria. In the presence of galactose, UDP-Gal is produced 879 and accumulated, which both decreases production of lipid II and produces low levels of 880 toxic lipid II*, which incorporates UDP-Gal in place of UDP-GlcNAc. Lipid II* is unable to 881 be properly polymerized into mature peptidoglycan and causes an "poisoning effect" in 882 peptidoglycan biosynthesis, which leads to eventual cell death. 


\section{Table 1. A suppressor screen yields identified mutations in additional putative}

885 targets that allowed reduced toxicity. A suppressor screen was performed to identify

886 additional genes or pathways involved in galactose associated toxicity. $\Delta$ galE cells with

887 a second copy of galK and galT (YC814) were grown overnight in LB containing 1\%

888 (w/v) galactose and allowed to incur natural mutations which reduced toxicity. Mutant

889 strains were enriched, verified, and the suppressor mutations identified by whole

890 genome sequencing. A selection of genes with suppressor mutations, the type of

891 mutations, amino acid sequence change, and gene information are described. fs, frame-

892 shift mutation; SNV, single nucleotide variation; MNV, multiple nucleotide variations.

\begin{tabular}{|c|c|c|c|c|}
\hline Gene & Type & Change & Protein function & Process \\
\hline$g t a B$ & SNV & R118P & UDP-glucose pyrophosphorylase & \multirow{4}{*}{$\begin{array}{l}\text { Central } \\
\text { metabolism }\end{array}$} \\
\hline araE & SNV & G31Y & L-arabinose permease & \\
\hline yvfH & MNV & Promoter & Lactate permease & \\
\hline ptsG & SNV & G329V & Glucose permease & \\
\hline tuaA & SNV & G9V & ABC transporter & \multirow{2}{*}{$\begin{array}{l}\text { Teichoic acid } \\
\text { production }\end{array}$} \\
\hline $\operatorname{tag} H$ & MNV & Promoter & ABC transporter & \\
\hline cypX & SNV & Promoter & Cytochrome P450 & $\begin{array}{l}\text { Pulcherrimin } \\
\text { production }\end{array}$ \\
\hline $\operatorname{con} B$ & SNV & N167S & Conjugation machinery & Conjugation \\
\hline oppC & SNV & Y178R & Oligopeptide $\mathrm{ABC}$ transporter & \multirow{2}{*}{$\begin{array}{l}\text { Oligopeptide } \\
\text { transport }\end{array}$} \\
\hline oppD & SNV & S208F & Oligopeptide ABC transporter & \\
\hline$g l c R$ & SNV & nt147fs* & Transcriptional repressor & \multirow{2}{*}{$\begin{array}{l}\text { Stress } \\
\text { response }\end{array}$} \\
\hline yrhJ & SNV & nt1046fs* & Cytochrome P450 & \\
\hline
\end{tabular}


A
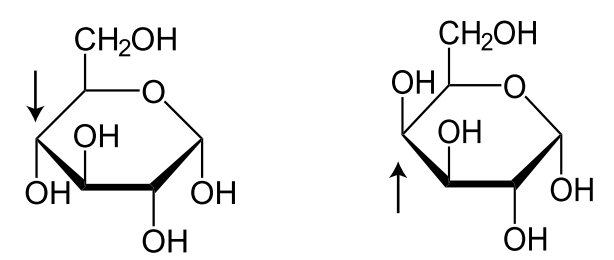

a-D-glucose

a-D-galactose

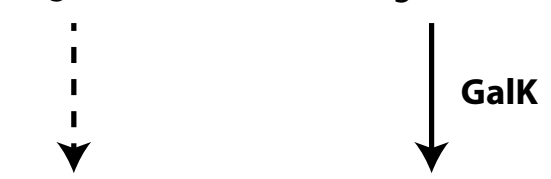

Glucose-1-P Galactose-1-phosphate

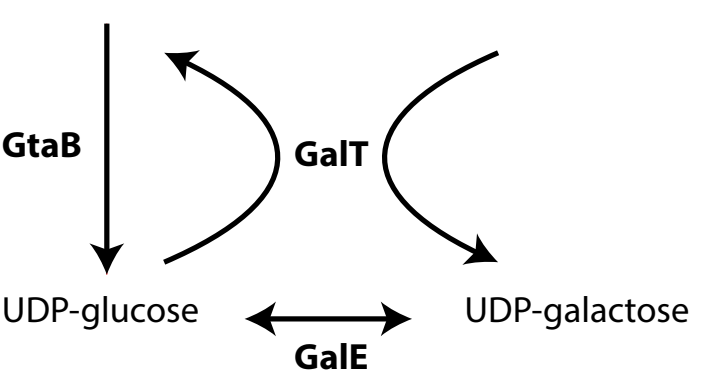

E

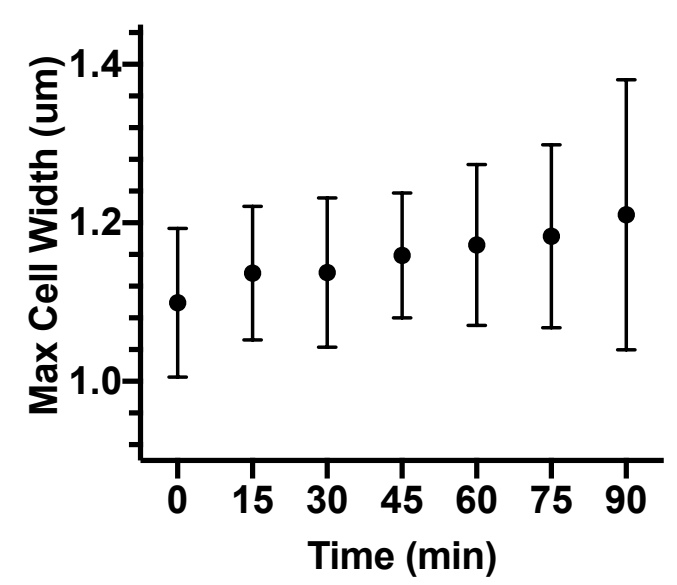

B

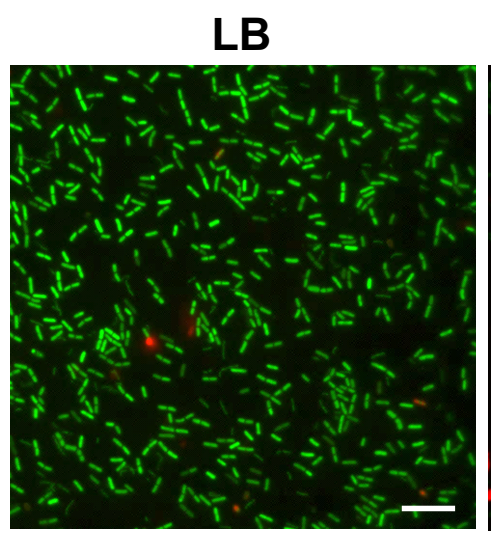

$\sim 1 \%$

C

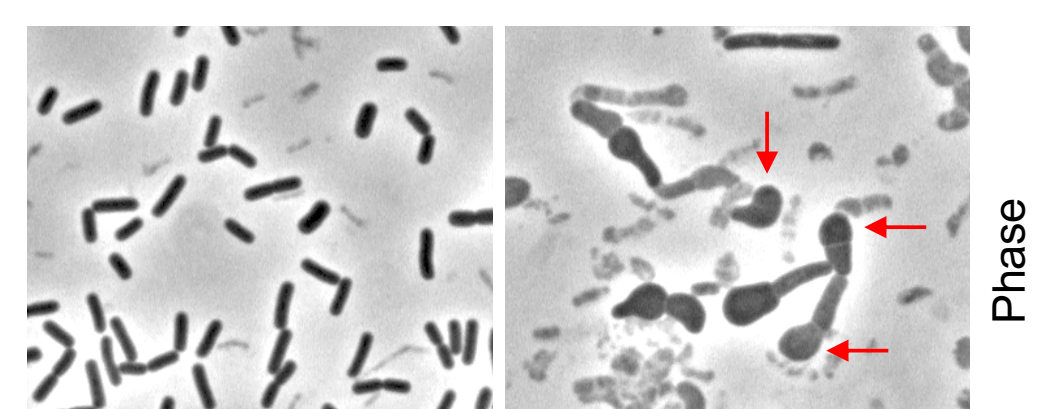

0
0
0
0
0
0

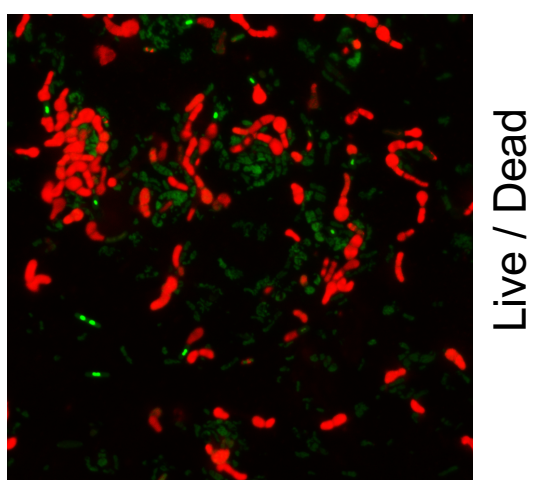

$\sim 98 \%$

$\mathscr{D}$
$\mathbb{\infty}$
$\frac{\mathbb{C}}{\alpha}$

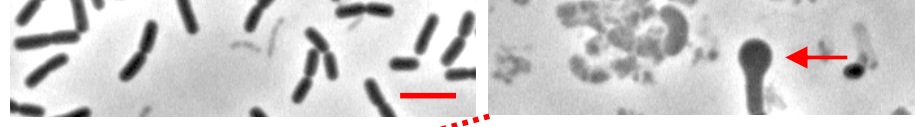

D
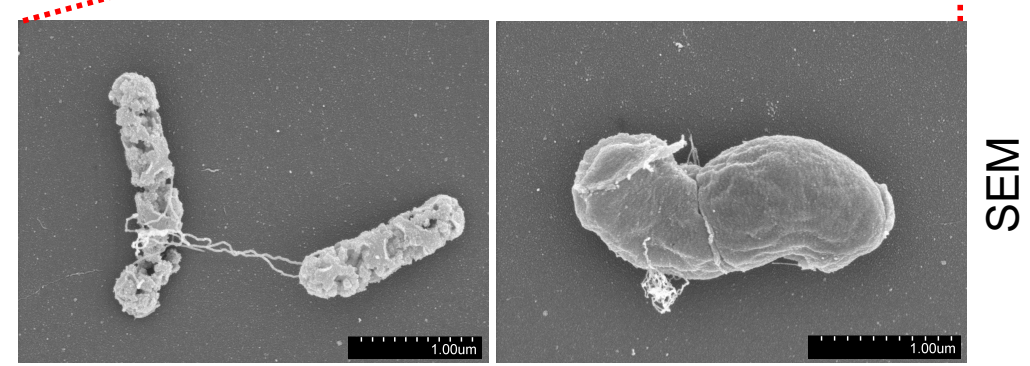

Figure 1 


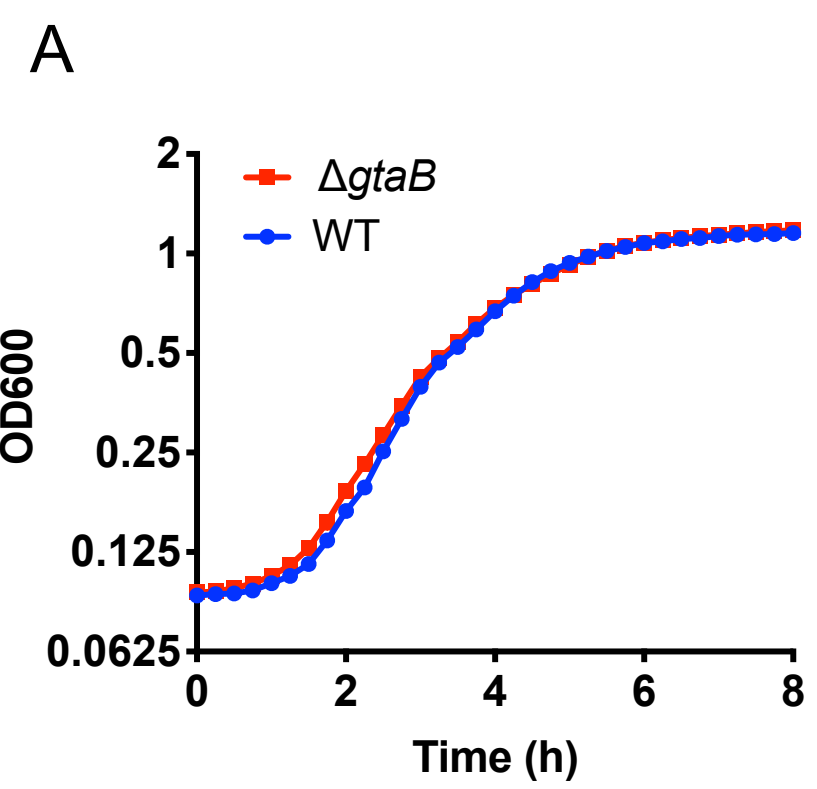

B

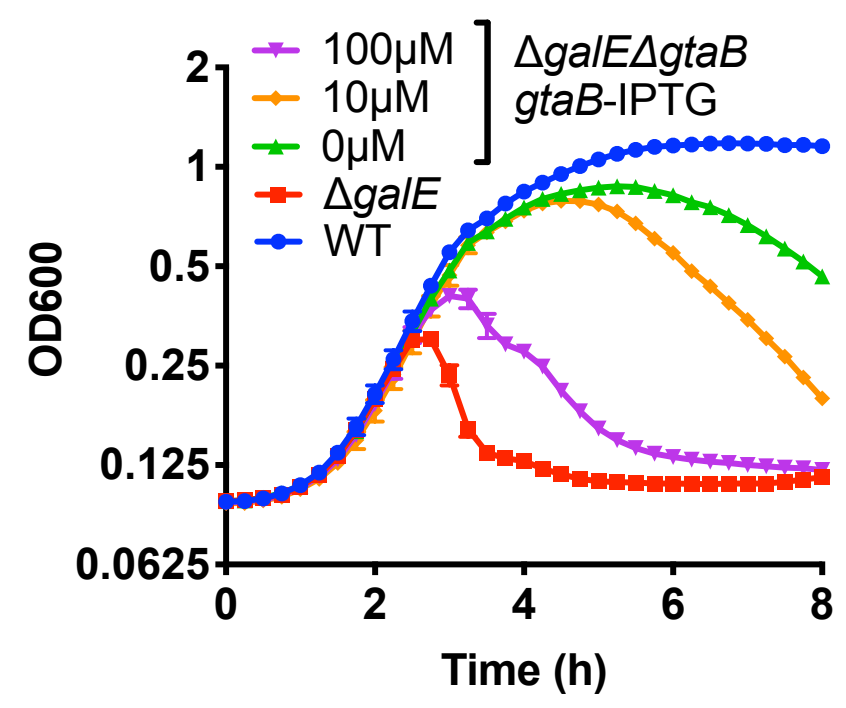

C Time (min)

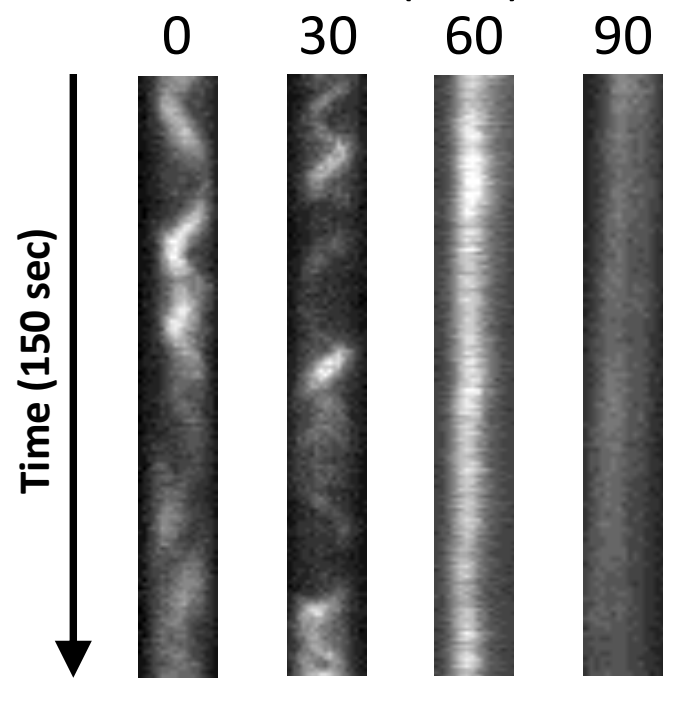

D

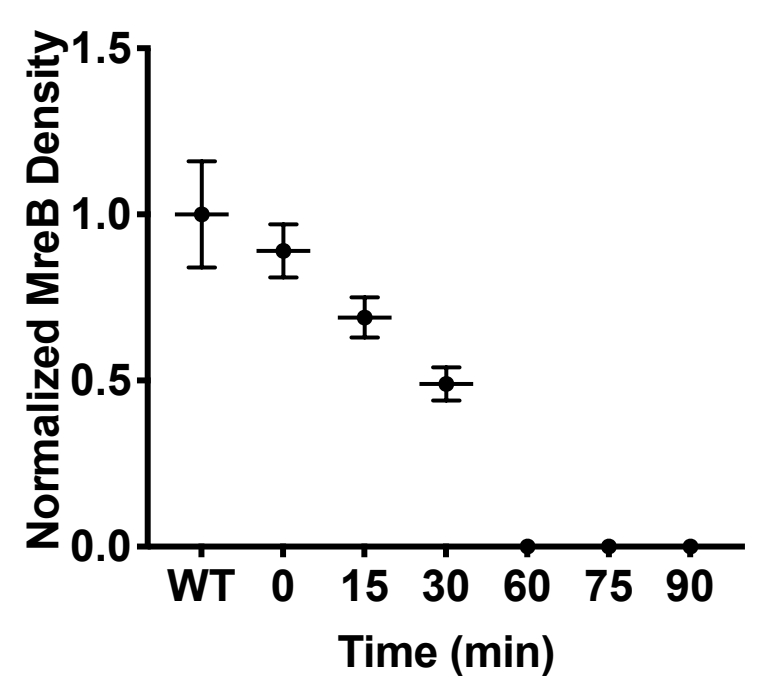

E Time (min)

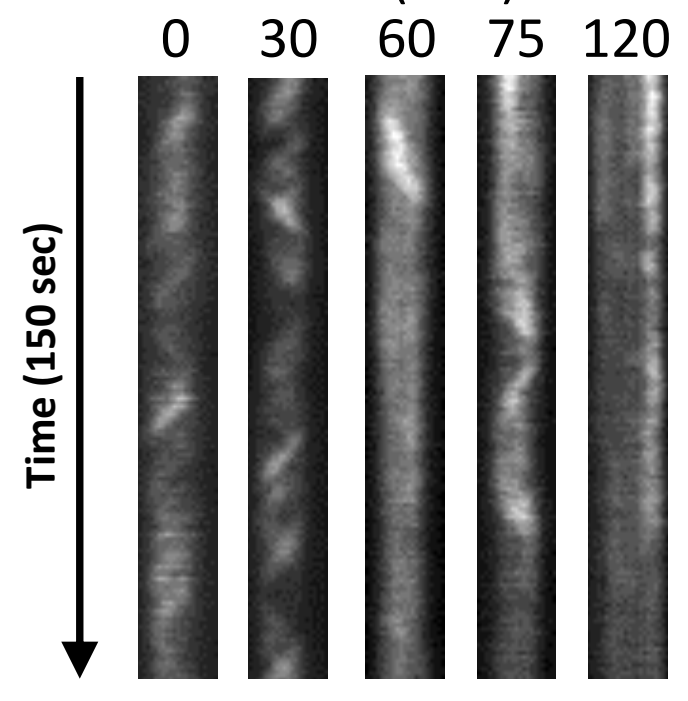

F

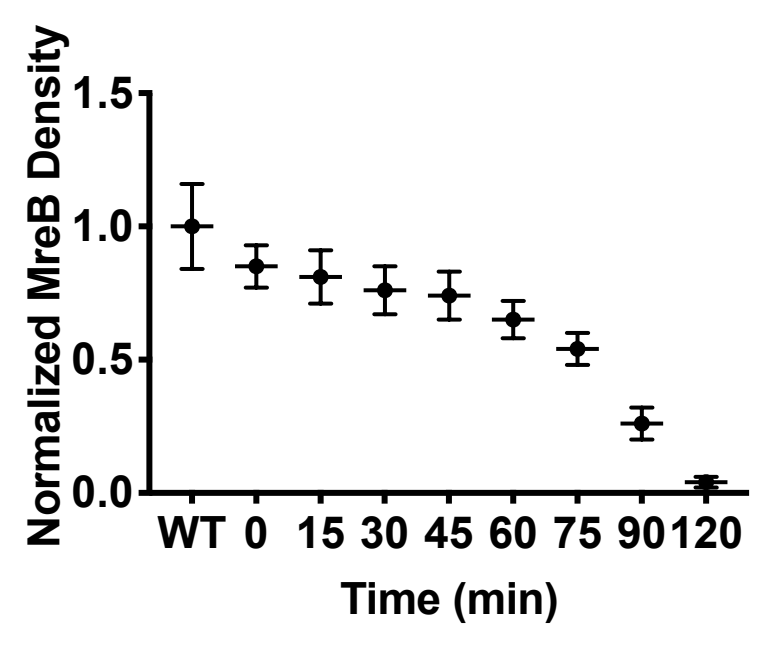

Figure 2 
A

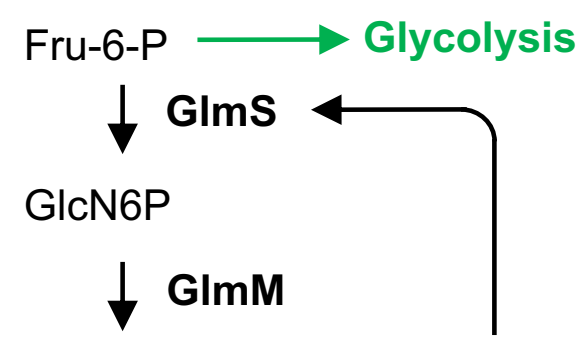

GIcN1P

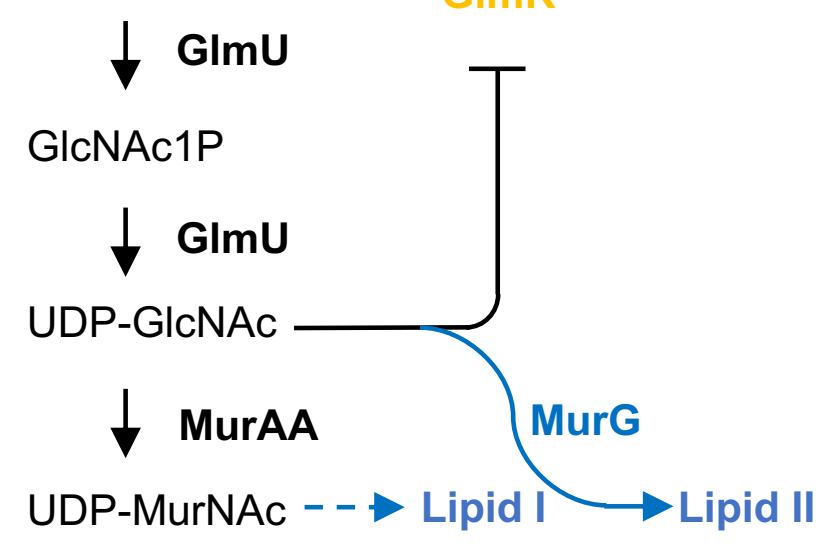

B

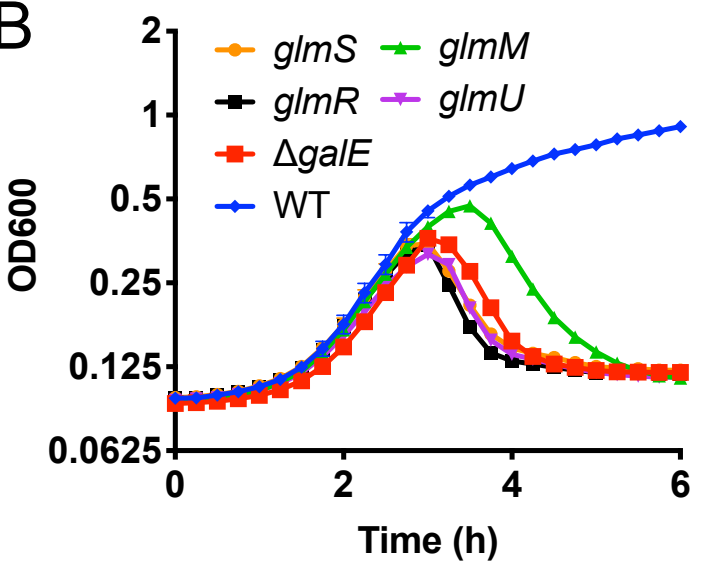

C

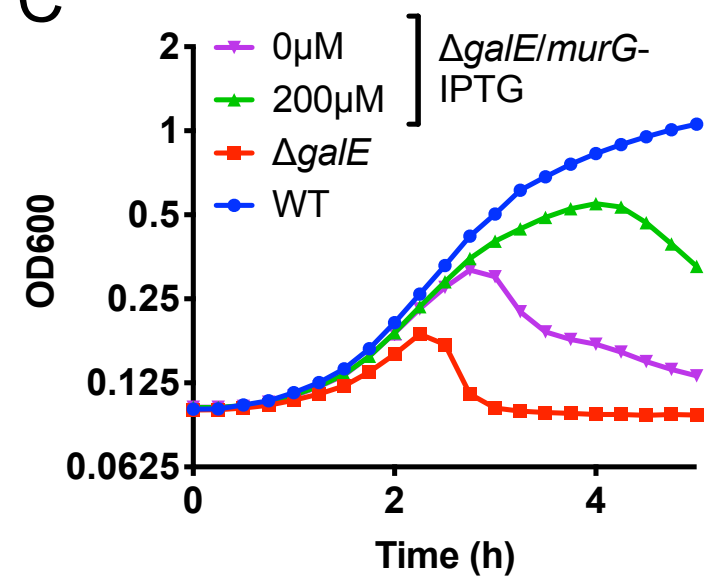

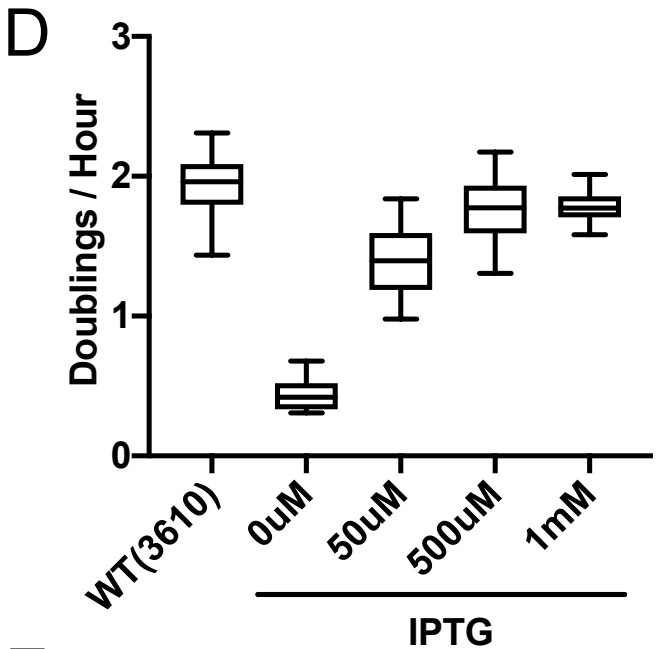

E

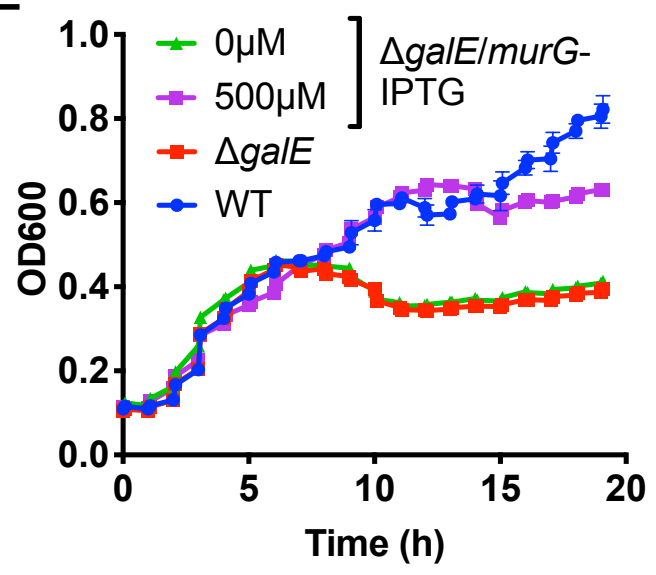

Figure 3 

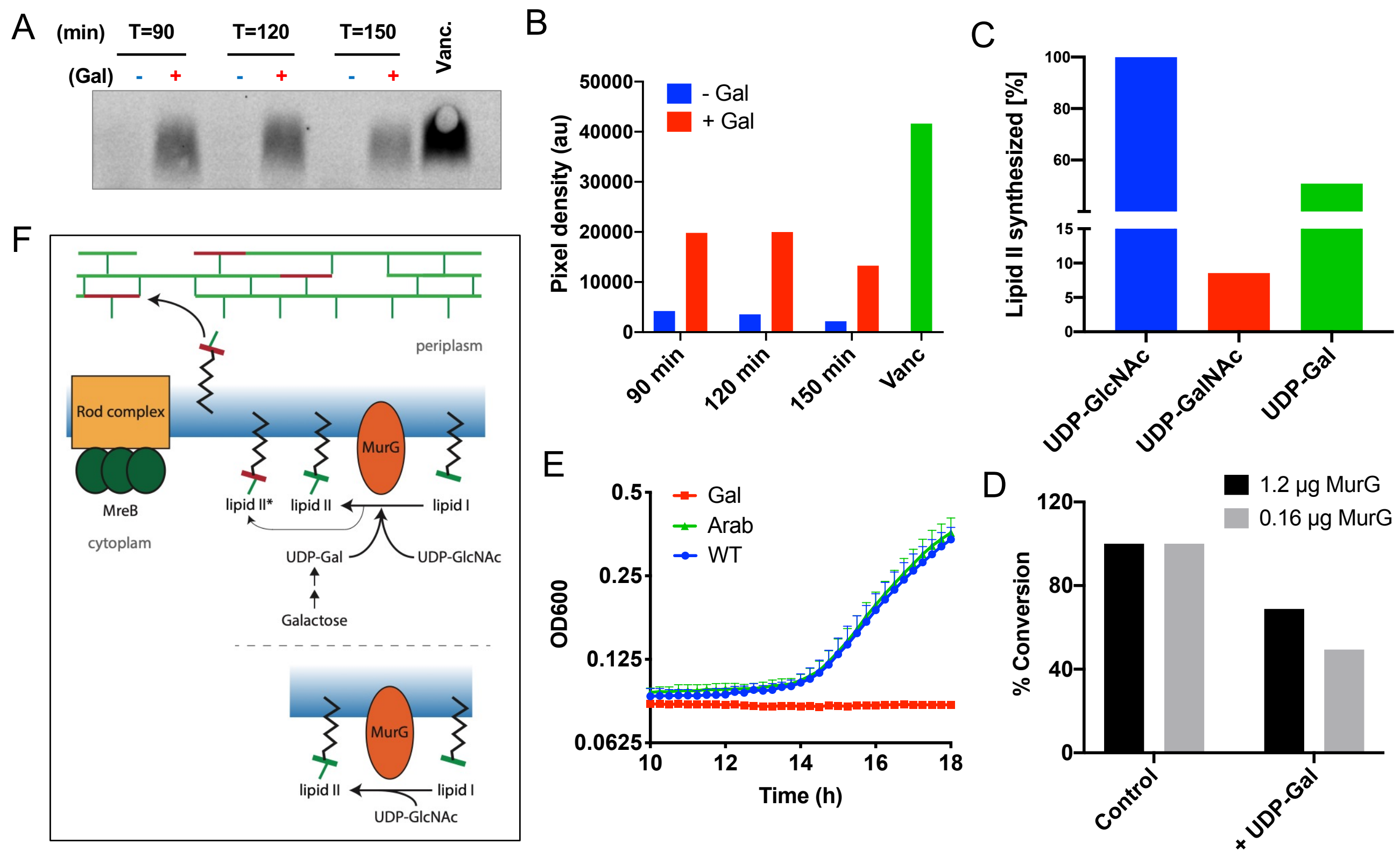

Figure 4 
A

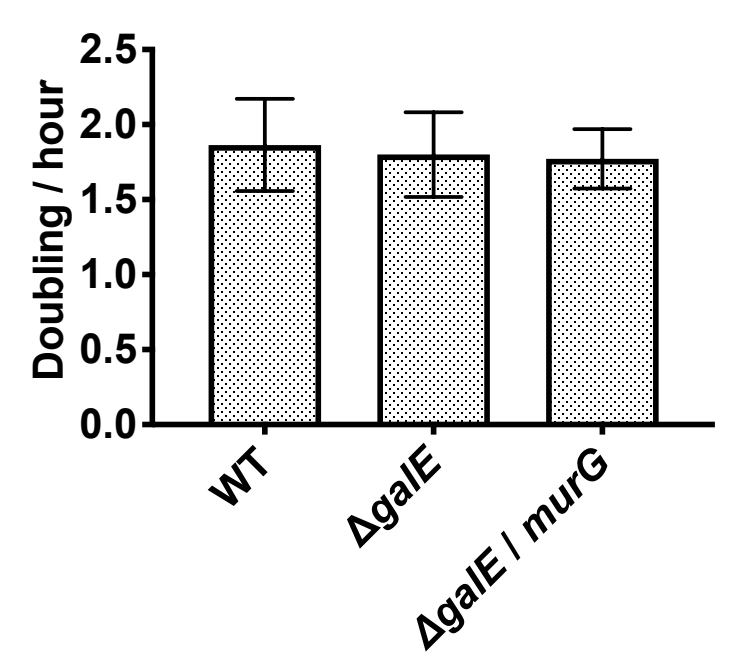

B

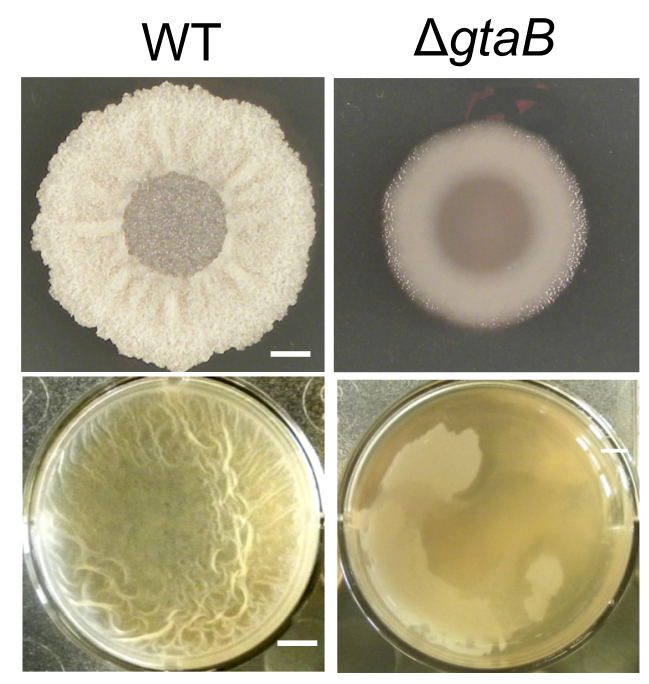

C
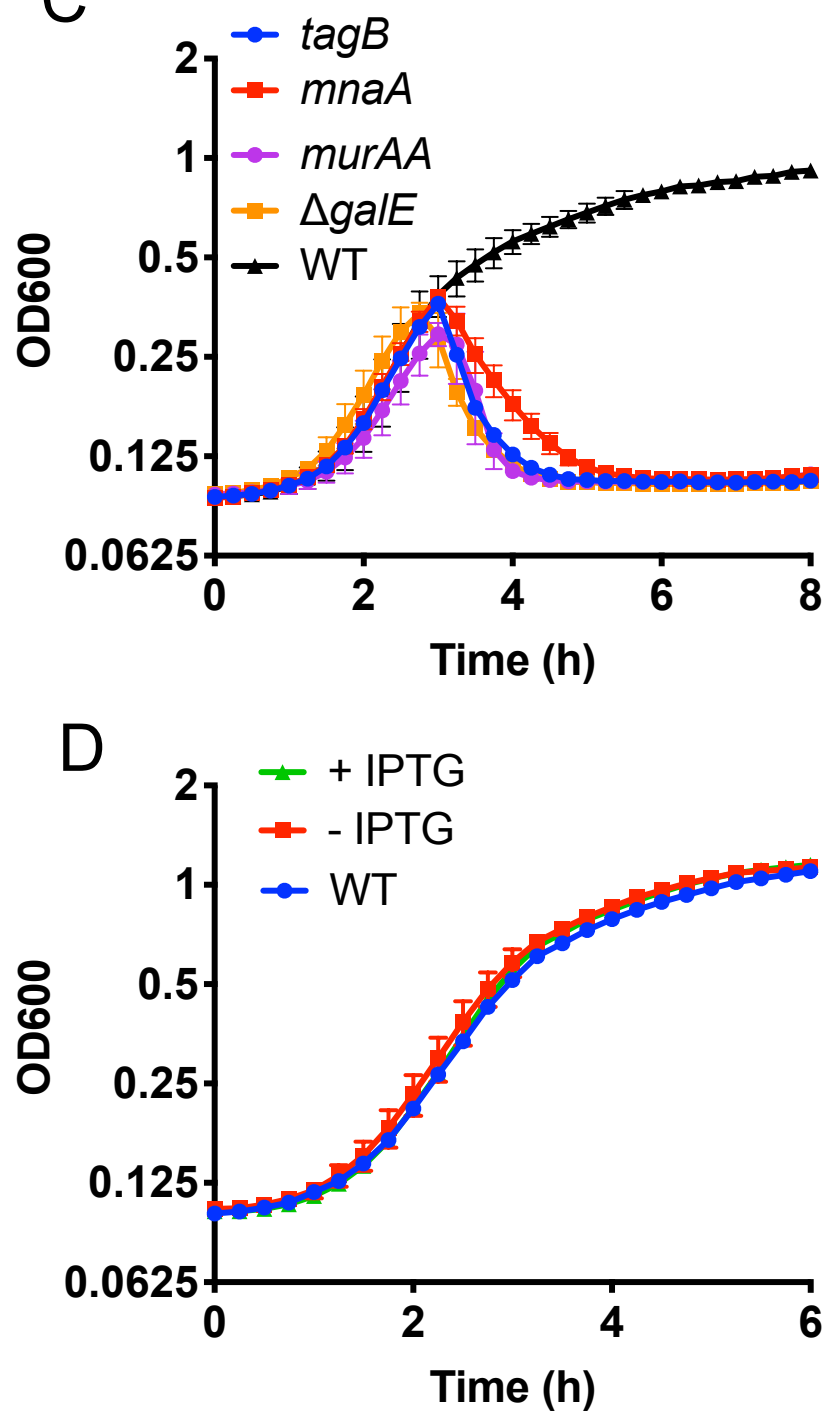

$E$

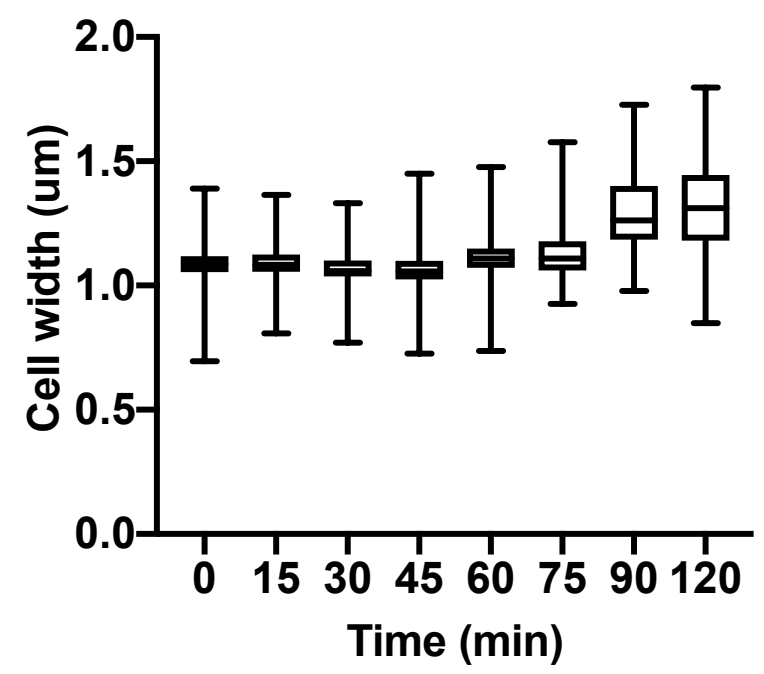



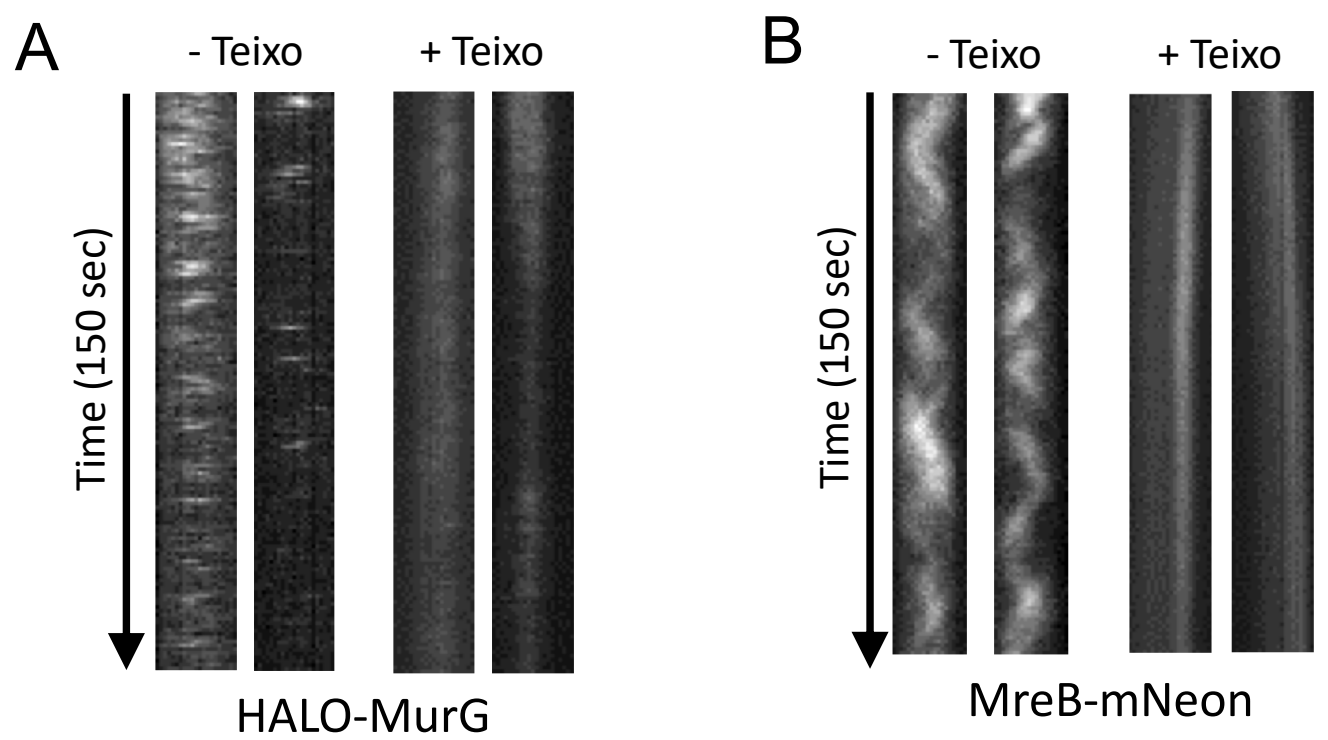

Supplemental Figure 2 


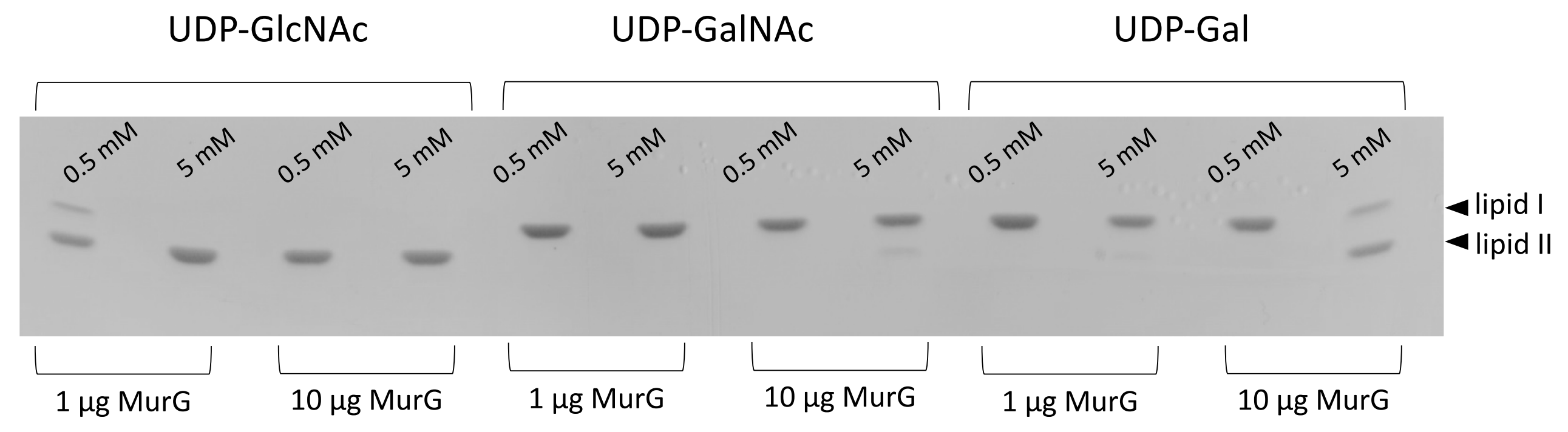




\section{Supplemental files_Habib et al.}

\section{Supplemental Methods}

4 Strain construction. General methods for molecular cloning followed published

5 protocols ${ }^{1}$. Long-flanking PCR mutagenesis was used to generate insertional deletion mutations ${ }^{2}$. To construct the $B$. subtilis $\triangle$ galE strain, genomic DNA was isolated from the strain YC814 and transformed into WT NCIB 3610 (hereafter 3610) and plated on

8 LB agar plates under tetracycline selection ${ }^{3}$. To construct the gtaB deletion strain (YC876), the insertional deletion mutation in gtaB was created by long-flanking PCR mutagenesis. The four primers (delta-gtaB-P1 to delta-gtaB-P4) used for gtaB PCR mutagenesis are listed in Table S2. The double mutant of $\Delta g a l E \Delta g t a B$ was made by introducing $\triangle$ galE::tet from YC814 to YC876 by transformation and selecting the transformants on LB plus both tetracycline and kanamycin. To construct an IPTG

14 inducible copy of gtaB, the native gtaB gene was amplified by PCR using primers $\mathrm{P}_{\text {gtaB- }}$

$15 \mathrm{~F} 1$ and $\mathrm{P}_{\mathrm{gtaB}}-\mathrm{R} 1$ and isolated 3610 genomic DNA as the template. The purified PCR

16 product was cloned into HindIII and Sall restriction sites of the plasmid pDR111. The

17 resulting plasmid was transformed into and purified from $E$. coli strain $\mathrm{DH} 5 \alpha$, verified by

18 DNA sequencing, and then transformed into the $B$. subtilis lab strain PY79.

19 Transformants were selected for double-crossover recombination at the chromosomal

20 amyE locus on LB agar plate with spectinomycin. The genomic DNA of successful

21 transformants was purified and used to transform the $\triangle$ galE $\Delta g t a B$ double mutant of $B$.

22 subtilis outlined above. The generation of IPTG inducible murG was performed as

23 above, amplified using primers $P_{\text {murG }}-\mathrm{F} 1$ and $P_{\text {murG }}-R 2$ and utilizing the HindIII and Nhel

24 restriction sites of pDR111. Similarly, the IPTG inducible copy of $\operatorname{tag} B$, murAA, and $m n a A$ were performed utilizing respective primers tagB-F1 and tagB-R1, murAA-F1 and

26 murAA-R1, and mnaA-F1 and mnaA-R1. To generate the $\Delta$ galE/mreB-mNeonGreen

27 reporter strain, genomic DNA from YC814 was isolated and transformed into bYS09, 28 containing a native promoter protein fusion of MreB-mNeonGreen ${ }^{4,5}$, and plated on LB

29 agar plate selecting for erythromycin resistance. To construct the $\Delta g a / E / m u r G / m r e B$ -

30 mNeonGreen strain, the pDR111-murG plasmid constructed above was transformed

31 into the $\Delta g a l E / m r e B-m N e o n G r e e n$ strain and selected for by plating on LB agar with 
32 spectinomycin. To generate IPTG inducible copies of $g / m U, g / m M, g / m S$, and $g / m R$,

33 genomic DNA from strains HB16910 (glmM), HB16951 (glmR), HB21922 (glmU), and

34 HB21942 (glmS), [gifts of the J. Helmann] were prepared and transformed into a $\Delta g a l E$

35 background selecting for chloramphenicol resistance.

The HALO-murG fusion (bYS993) was generated by transforming PY79 with a Gibson assembly consisting of five fragments: 1) PCR with primers oMD191 and oMD108 and PY79 template genomic DNA; 2) PCR with primers oJM28 and oJM29 and pWX467 [gift of D. Rudner] template genomic DNA (containing the kanamycinresistance cassette loxP-kan-loxP); 3) PCR with primers oMD234 and oMD232 and template pDR150 [gift of D. Rudner] genomic DNA; 4) PCR with primers oYS600 and oAB60 and HALO-tag template; 5) PCR with primers oYS835 and oMD197 and template PY79 genomic DNA. mreB-mNeonGreen fusion in a galE deletion background (bYS992) was generated by transforming bYS09 with genomic DNA from GalE KO::erm. bYS995 [amyE::Phyperspank-HALO-murG::kan, galE::erm] was generated by transforming bYS993 with genomic DNA from galE::erm.

The amyE::Phyperspank-mraY::erm (bYS424) was generated by transforming PY79 with a Gibson assembly consisting of five fragments: 1) PCR with primers oMD191 and oMD108 and PY79 template genomic DNA; 2) PCR with primers oJM28 and oJM29 and pWX467 [gift of D. Rudner] template genomic DNA (containing the erythromycin -resistance cassette loxP-erm-loxP); 3) PCR with primers oMD234 and oMD232 and template pDR150[gift of D. Rudner] genomic DNA; 4) PCR with primers oYS682 and oYS357 and PY79 template; 5) PCR with primers oMD196 and oMD197 and template PY79 genomic DNA.

bYS997 [mreB-mNeonGreen, amyE::Phyperspank-murG::spec, galE::erm] was generated by transforming bYS992 with genomic DNA from amyE::PhyperspankmurG::spec. bYS998 [mreB-mNeonGreen, amyE::Phyperspank-MurAA::spec,

61 galE:::erm] was generated by transforming bYS992 with genomic DNA from CH125

62 (bCH125). bYS999 [mreB-mNeonGreen, amyE::Phyperspank-MraY::erm, galE::erm] 
was generated by transforming bYS992 with genomic DNA from amyE::PhyperspankmraY::erm (bYS424).

Overexpression of the native E. coli murG was performed by isolating genomic DNA from the E. coli lab strain DH5 $\alpha$ and amplifying native murG gene utilizing primers $\mathrm{P}_{\text {murG }}-\mathrm{F} 2$ and $\mathrm{P}_{\text {murG }}-\mathrm{R} 2$. The resulting purified PCR product was cloned into pUC18 plasmid utilizing restriction sites BamHI and Pstl. The recombinant plasmid was transformed into and purified from $\mathrm{DH} 5 \alpha$ and sequence verified. The resulting plasmid was transformed into the E. coli strain JW0742 ( $\Delta$ galE, the Keio collection) ${ }^{6}$, and selected for by plating on LB agar plate with ampicillin. B. subtilis 168 murG was amplified using primers murGBsub FW and RV and cloned into pET21b vector (Novagen) by InFusion cloning to generate a C-terminal His6 fusion protein. The recombinant plasmid was transformed into $\mathrm{DH} 5 \alpha$, verified by DNA sequencing, and then transformed into BL21/DE3 for protein expression. Expression of MurGBsub-His6 and MurGSaur-His6 and purification were performed as described ${ }^{7}$. frame deletion of galE (VCA0774) was constructed by cloning the regions flanking target genes into the suicide vector pWM91 containing a sacB counter-selectable marker ${ }^{9}$ utilizing primers galE-Up-Xhol-5, galE-Up-3, galE-Down-5, and galE-DownNotl-3. Double-crossover recombination mutants were selected using $10 \%$ sucrose plates and confirmed via PCR. Complementation of murG was constructed by cloning the complete murG (VC2401) sequence into the pSRKTc vector utilizing primers murGNdel-5' and murG-Xbal-3' 10. For in vitro growth experiments, cells from overnight cultures grown in LB medium of the WT, the galE mutant and murG complemented mutant were inoculated 1:100 into fresh LB medium supplemented with tetracycline of 2 $\mu \mathrm{g} \mathrm{mL}^{-1}$ and IPTG (isopropyl-beta-D-thiogalactopyranoside) of $0.5 \mathrm{mM}$ at a final concentration, when indicated, $0.05 \%$ galactose was included, statically grown at $37^{\circ} \mathrm{C}$ and measured $\mathrm{OD}_{600}$ using Bioscreen C (Type FP-1100-C) instrument at time points

92 indicated. 
94 Whole genome sequencing. To prepare DNA for whole genome sequencing, all

95 strains were grown to a mid-exponential phase $\mathrm{OD}_{600}$ of 0.4 and $1 \mathrm{~mL}$ of culture spun

96 down 2 minutes at $16,000 \mathrm{~g}$. Cells were resuspended in $450 \mu \mathrm{L}$ of dd $\mathrm{H}_{2} \mathrm{O}$ with $50 \mu \mathrm{L}$

$97500 \mathrm{mM}$ EDTA and $60 \mu \mathrm{L}$ lysozyme at $20 \mathrm{mg} / \mathrm{mL}$, and incubated for 30 minutes at

$9837^{\circ} \mathrm{C}$. After incubation, $650 \mu \mathrm{L}$ of cell lysis solution (Promega) was added and tube was

99 inverted several times, followed by $250 \mu \mathrm{L}$ of protein perception solution (Promega).

100 Tube was vortexed until homogenous and centrifuged for $5 \mathrm{~min}$ at $16,000 \mathrm{~g} .900 \mu \mathrm{L}$ of

101 supernatant was transferred to a new tube and $600 \mu \mathrm{L}$ isopropanol added to precipitate

102 DNA, with the tube inverted several times and incubated at room temperature for 2 min,

103 then spun $16,000 \mathrm{~g}$ for $5 \mathrm{~min}$. DNA pellet was washed in $70 \%$ ethanol and spun down at

$10416,000 \mathrm{~g}$. Tube was dried at room temperature under air flow for 10 minutes and then

105 resuspended in $100 \mu \mathrm{L}$ of $\mathrm{ddH}_{2} \mathrm{O} .2 \mu \mathrm{g}$ of DNA was digested for 30 min with dsDNA

106 Fragmentase (NEB) and then stopped with EDTA. DNA fragments of approximately

107 200-bp were selected for using AMPure XP beads (Beckman Coulter, Indianapolis, IN)

108 according to manufacturer protocols. Resulting fragments were prepared and labeled

109 for whole genome sequencing using NEBNExt Ultra DNA Library Prep Kit (NEB) from

110 Illumina technologies. Determination of resulting DNA library purity and concentration,

111 and subsequent sequencing was performed by the Harvard Bauer Core Facility

112 (Harvard, Cambridge, MA).

113

114

115

116

117

118

119

120 


\section{Supplemental Figure legends}

122 Fig. S1. (A) To determine if any difference in growth rate was apparent at a single cell

123 resolution upon deletion of galE, the same strains were grown on a $\mathrm{CH}$ media agar pad

124 and imaged over time. Similar to OD600 measurements, no change in rate was

125 observed. Bars indicate standard deviation. Colony and pellicle biofilm development by

126 the WT (3610) and the $\triangle g t a B$ mutant (YC876) in solid and liquid MSgg media. The

$127 \Delta g t a B$ mutant was unable to form robust biofilms, presumably due to lack of the

128 essential nucleotide sugar UDP-Glc for making exopolysaccharides of the biofilm

129 matrix. Biofilms were incubated at $30^{\circ} \mathrm{C}$ for 3 days before imaging. Scale bars indicate 1

$130 \mathrm{~mm}$, pellicle well diameter is $3.5 \mathrm{~cm}$. (C) To determine if the rescue exhibited by murG

131 was unique to murG or shared among other glycotransferases which utilize UDP-NAG,

132 an IPTG inducible copy of murAA ( $\mathrm{CH} 125)$, glms (CH127), tagB (CH123), and mnaA

133 (CH129) were each respectively introduced into the $B$. subtilis $\Delta$ galE mutant and

$134(\mathrm{CH} 062)$ and grown in LBga shaking at $37^{\circ} \mathrm{C}$ with $0.5 \mathrm{mM}$ IPTG, along with wild type

135 and $\Delta$ galE strains, and OD600 measurements taken every 15 minutes. Overexpression

136 of genes had no rescue associated with it as compared to $\Delta$ galE strain. (D) To

137 determine if the rescue seen by murG overexpression was due to an increase in growth

138 rate, the $\Delta$ galE/PTG-murG strain created above as well as wild type was grown

139 shaking at $37^{\circ} \mathrm{C}$ in LB without (-) and with (+) $500 \mu \mathrm{M}$ of IPTG with OD600

140 measurements taken every 15 minutes. Overexpression of murG had no effect on

141 growth rate. (E) The effect of MurG overexpression on toxicity as a function of maximal

142 cell width of $\sim 100$ cells using microscopy. Cells were grown in galactose and MurG

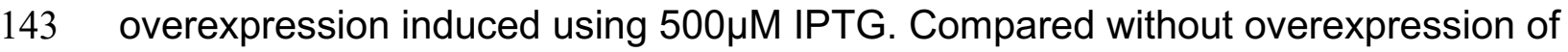

144 MurG, cells were able to maintain a consistent max cell width for a greater amount of

145 time, indicating a rescue in toxicity resulting from MurG. Error bars represent standard

146 deviations.

148 Fig. S2. (A) B. subtilis $\triangle$ galE mutant expressing a HALO-MurG fusion was imaged

149 using TIRF microscopy on a $\mathrm{CH}$ agar pad with or without the addition of $25 \mathrm{ug} / \mathrm{mL}$ of

150 teixobactin. Kymographs were created utilizing the images obtained. It was found that

151 upon addition of teixcobactin, activity of MurG was eliminated, suggesting that the 
152 diffusion of MurG could serve as a reporter for its activity. As a control, the B. subtilis

$153 \Delta$ SgalE mutant expressing the MreB-mNeonGreen fusion was similarly imaged with and

154 without the addition of $25 \mathrm{ug} / \mathrm{mL}$ of teixobactin. Upon addition, MreB diffusion was also

155 arrested, providing additional support for the use of movement as a measurement for

156 protein activity.

158 Fig. S3. One or ten microgram (ug) of B. subtilis MurG was mixed with either 0.5 or 5 $159 \mathrm{mM}$ of UDP-GIcNAc, UDP-GaINAc, or UDP-Gal (respectively) to determine the ability of 160 MurG to utilize these substrates in the generation of lipid II from lipid I. The products of 161 the reactions were run on TLC and quantified, showing that MurG can utilize both UDP-

162 GalNAc as well as UDP-Gal as substrates, though at a reduced efficiency to the

163 preferred substrate of UDP-GIcNAc

Supplemental movie 1. The B. subtilis $\triangle$ galE mutant expressing a MreB-mNeonGreen fusion protein in native locus was imaged via TIRF microscopy after the addition of

167 galactose $(0.5 \%$, w/v) onto a $\mathrm{CH}$ agar pad. By 60 minutes after galactose addition, most

168 of MreB filaments completely ceased movement or were depolymerized, indicating

169 inhibition of cell wall biosynthesis in cells exhibiting strong toxicity. Cells were imaged at 1701 second intervals with an exposure time of $300 \mathrm{~ms}$.

172 Supplemental movie 2. The B. subtilis $\triangle$ galE mutant expressing a MreB-mNeonGreen

173 fusion protein in native locus and Phyperspank MurG in AmyE locus was imaged via

174 TIRF microscopy after the addition of galactose $(0.5 \%$, w/v) and $1 \mathrm{mM}$ IPTG onto a $\mathrm{CH}$

175 agar pad. By 90 minutes after galactose addition, directional motion of MreB filaments

176 could still be observed. Cells were imaged at 1 second intervals with an exposure time

177 of $300 \mathrm{~ms}$.

179 Supplemental movie 3. The B. subtilis $\triangle$ galE mutant expressing a MreB-mNeonGreen

180 fusion protein in native locus and Phyperspank MurAA, MraY or MurG in AmyE locus

181 was imaged via TIRF microscopy after the addition of galactose $(0.5 \%, w / v)$ and $1 \mathrm{mM}$ 
182 IPTG onto a $\mathrm{CH}$ agar pad. After 90 minutes galactose addition, directional motion of

183 MreB filaments could only be observed for cells with MurG overexpression but not for

184 cells with MurAA or MraY overexpression. Cells were imaged at 1 second intervals

185 using TIRF microscopy with an exposure time of $300 \mathrm{~ms}$.

187 Supplemental movie 4. B. subtilis $\triangle$ galE mutant expressing a HALO-MurG fusion was

188 imaged using TIRF microscopy on a $\mathrm{CH}$ agar pad without or with the addition of

189 teixobactin (2.5 and $25 \mathrm{ug} / \mathrm{mL})$. Cells were imaged at 1 second intervals using TIRF

190 microscopy with an exposure time of 500ms.

192 Supplemental movie 5. B. subtilis expressing a MreB-mNeonGreen fusion was imaged

193 using TIRF microscopy on a $\mathrm{CH}$ agar pad without or with the addition of teixobactin (2.5

194 and $25 \mathrm{ug} / \mathrm{mL})$. Cells were imaged at 1 second intervals with an exposure time of

\section{$195300 \mathrm{~ms}$.}

197 Supplemental movie 6. To determine the effect of UDP-Gal accumulation on MurG in 198 vivo, the $B$. subtilis $\triangle$ galE mutant expressing a HALO-MurG fusion was imaged via TIRF 199 microscopy 60,90 , and 120 minutes after the addition of galactose $(0.5 \%$, w/v) onto a $200 \mathrm{CH}$ agar pad. After 90 minutes after galactose addition, a significant population of MurG 201 had arrested movement, indicating that UDP-Gal accumulation causes an inhibition of 202 MurG activity. Cells were imaged at 1 second intervals with an exposure time of 500ms. 
bioRxiv preprint doi: https://doi.org/10.1101/2021.01.22.426804; this version posted January 22, 2021. The copyright holder for this preprint

(which was not certified by peer review) is the author/funder. All rights reserved. No reuse allowed without permission.

Table S1: Strains used in this study.

\section{Strains}

B. subtilis PY79

B. subtilis NICB3610

$\mathrm{CH} 062$

$\mathrm{CH} 112$

$\mathrm{CH} 117$

$\mathrm{CH} 123$

$\mathrm{CH} 125$

$\mathrm{CH} 127$

$\mathrm{CH} 129$

$\mathrm{CH} 143$

$\mathrm{CH} 150$

$\mathrm{CH} 151$

$\mathrm{CH} 152$

$\mathrm{CH} 153$

YC814

YC876

$\mathrm{DH} 5 \alpha$

VCA0774

VC2401

bYS09

bYS424

bYS992

bYS997

bYS998

bYS999

bYS993

bYS995

\section{Genotypes}

A laboratory strain for genetic manipulation

Undomesticated $B$. subtilis strain capable of biofilm formation $\triangle$ galE::tet ${ }^{\mathrm{R}}$ in 3610

amyE:: $\mathrm{P}_{\text {hyspank-murG, } \Delta \text { galE::tet }}{ }^{\mathrm{R}}$ in 3610

$\triangle$ gtaB::spec ${ }^{R}, \Delta$ galE::tet ${ }^{R}$, amyE::P $P_{\text {hyperspank }}-g t a B$ in 3610

$\triangle$ galE::tet ${ }^{\mathrm{R}}$, amyE:: $\mathrm{P}_{\text {hyspank }}$-tagB::spec ${ }^{\mathrm{R}}$ in 3610

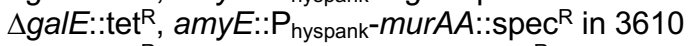

$\triangle$ galE::tet ${ }^{R}$, amyE::P hyspank-gImS::spec $^{R}$ in 3610

$\Delta$ galE::tet ${ }^{\mathrm{R}}$, amyE:: $\mathrm{P}_{\text {hyspank }}-m n a A::$ spec $^{\mathrm{R}}$ in 3610

$\triangle$ galE::kan ${ }^{\mathrm{R}}$, pUC18-murG in DH5a

$\triangle$ galE::tet ${ }^{R}$, amyE::Physpank-gImU

$\Delta$ galE::tet ${ }^{\mathrm{R}}$, amyE::P $\mathrm{P}_{\text {hyspank }}-\mathrm{glmM}$

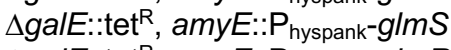

$\triangle$ galE::tet ${ }^{\mathrm{R}}$, amyE::P hyspank-gImR

$\Delta$ galE::tet ${ }^{R}$, amyE::P hyspank-galT-galK::spec $^{R}$ in 3610

$\Delta g t a B:: k^{R}{ }^{R}$ in 3610

A laboratory strain of $E$. coli

$\triangle$ galE::tet ${ }^{\mathrm{R}}$ in V. cholerae E1 Tor $\mathrm{C6706}$

$\triangle$ galE::tet ${ }^{R}$, pSRKTc-murG in V. cholerae E1 Tor C6706

mreB-mNeonGreen

amyE::Phyperspank-mraY:;erm

mreB-mNeonGreen, $\triangle$ galE::erm

mreB-mNeonGreen, $\Delta$ galE::erm, amyE::Phyperspank-murG::spec

mreB-mNeonGreen, $\Delta$ galE::erm, amyE::P $P_{\text {hyperspank-murAA::spec }}$

mreB-mNeonGreen, $\Delta$ galE::erm, amyE::Phyperspank-mraY::erm

amyE::P $P_{\text {hyperspank-HALO-murG::kan }}$

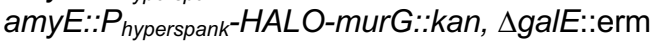

References

11

12

this study

this study

this study

this study

this study

this study

this study

this study

this study

this study

this study

this study

13

this study

Invitrogen

this study

this study

5

this study

this study

this study

this study

this study

this study

this study 
Table S2: Oligonucleotides used in this study.

\section{Name}

PgtaB-F1

PgtaB-R1

murG-F1

murG-R1

murG-F2

murG-R2

murGP-F1

murGP-F1

glmS-F1

glmS-R1

mnaA-F1

mnaA-R1

tagB-F1

tagB-R1

murAA-F1

murAA-R1

Delta/gtaB-P1

Delta/gtaB-P2

Delta/gtaB-P3

Delta/gtaB-P4

murG-NdeI-5

murG-XbaI-3

galE-Up-XhoI-5

galE-Up-3

galE-Down-5

galE-Down-NotI-3

oMD191

oMD108

oJM029

oJM028

oMD234

oMD232

oYS357

oYS600

oYS682

oAB60

oYS835

oMD196

oMD197

oMD349

oMD350

oMD351

oMD352

oMD353

oMD354

pET21b FW

pET21b RV

murGBsub FW

murGBsub RV
Sequence (5'-3')

GTAAAGCTTAAGGAGGAACTACTATGAAAAAAGTACGTAAAGCCA

GTAGTCGACTTAGATTTCTTCTTTGTTTAGTAA

GTACAAGCTT AAGGAGGAACTACT ATGCGAATTGCAATAAGC

GTACGCTAGCCTATTTTTTTAATTCCTC

GACTGGATCCGATGCTGTTGCGTATTGA

GACTCTGCAGCGCAGTTTTGCCAATTGT

TAACCATGGGTCGAATTGCAATAAGCG

GACCGGATCCCCTTTTTTTAATTCCTCGAGTACGC

GTACAAGCTTAAGGAGGAACTACTATGTGTGGAATCGTAGGTTA

GTACGCTAGCTTACTCCACAGTAACACTCTTCG

GTACAAGCTTAAGGAGGAACTACTATGAAAAAACTAAAAGTGAT

GTACGCTAGCTTATTTGCCTGTAAATGAATCCG

GTACAAGCTTAAGGAGGAACTACTATGAAAATAAGATCACTACT

GTACGCTAGCTTAGCTTATTAAATTTTCGATGAAAT

GTACGTCGACAAGGAGGAACTACTATGGAAAAAATCATCGTCCG

GTACGCTAGCTTATGCATTTAAGTCAGAAACGACTTCTTTA

TCTGCGGATGGCCTTAAACATGT

CAATTCGCCCTATAGTGAGTCGTGGCTTTACGTACTTTTTTCAT

CCAGCTTTTGTTCCCTTTAGTGAGCTAAACAAAGAAGAAATCTAA

CATTTTAAACTTGATTATTATGA

GGGAATTCCATATGATGAACAAGAATAAAAAATTAATGGTGATG

CTAGTCTAGATTATTCGGTAATGGCGATGAT

CCGCTCGAGAAGACTTAACTGAGCACGAT

CAAAAATACGTCGAGTAATTTTTCTCTCTCCAT

AGAAAAATTACTCGACGTATTTTTGCTCAGTCA

ATAAGAATGCGGCCGCTAACCCTGCCGTCACTATCG

TTTGGATGGATTCAGCCCGATTG

ACGAACGGTAGTTGACCAGTGCTCCCTGTCTTGACA

CTCCTTATTTGATTTTTTGAAGAC

CAGGGAGCACTGGTCAAC

TTCTGCTCCCTCGCTCAG

ATACGAACGGTACTGAGCGAGGGAGCAGAATAATGGATTTCCTTACGCGAAATACG

GGTAGTTCCTCCTTAAAGCTTAATTGTTATCCGCTCACAAT

TCTTTCGGTAAGTCCCGTCTAGCCTTGCCCTTATAACCACACCTCGATGTAAATTCCTAA GAGCGGATAACAATTAAGCTTTAAGGAGGAACTACCATGGCAGAAATCGGTACTGGC GGATAACAATTAAGCTTTAAGGAGGAACTACCATGCTTGAGCAAGTCATTCTGTTTAC

GCCGCTTCCTTGGCCTGAGC

CAGGGACCGGGCTCAGGCCAAGGAAGCGGCATGCGAATTGCAATAAGCGG

GGGCAAGGCTAGACGGG

TCACATACTCGTTTCCAAACGGATC

GGGTGTCGATTGCTTTGACTAC

TACGAACGGTAGTTGACCAGTGCTCCCTGTTCTCTATAA

CATAACGATAACCCTGTTTTG

ATACGAACGGTACTGAGCGAGGGAGCAGAAGGAAACAATTGATGC

GATAACCCTGTCCAACAAAACAGGGTTATCGTTATGTTATAGAGA

AACTTAGACTGGGGGAAAAAAGAAATGGCACACATCGTAATGGTAG

AGATCCAGGACCTTGTCCGCTACCCTCAAGTTTGTAAAGT

TCATCCATTCCATGCG

CTTGAGGGTAGCGGACAAGGTCCTGGATCTGGTCAAGGCAGTGGGTCAC

CTGGTAGCGGCCAAGGCCCTGGATCTGGACAAGGCAGCGGCCCTGGACAA

GGATCTGGCCCAGGTCAAGGCATGCGAATTGCAATAAGCGGAG

CATCGTTGTATGATTCGCGAGC

CACCACCACCACCACCACT

ATGTATATCTCCTTCTTAAAGTTAA

GAAGGAGATATACATATGCGAATTGCAATAAGCGGAGG

GTGGTGGTGGTGGTGTTTTTTTAATTCCTCGAGTACGC 
2991 Sambrook, J. \& Russell, D. W. Molecular Cloning. A Laboratory Manual. . (Cold Spring

$300 \quad$ Harbor Laboratory Press, 2001).

3012 Wach, A. PCR-synthesis of marker cassettes with long flanking homology regions for gene sidruptions in Saccharomyces cerevisiae. Yeast 12, 259-265 (1996). Gryczan, T. J., Contente, S. \& Dubnau, D. Characterization of Staphylococcus aureus plasmids introduced by transformation into Bacillus subtilis. J. Bacteriol. 134, 318-329 (1978).

4 Schirner, K. et al. Lipid-linked cell wall precursors regulate membrane association of bacterial actin MreB. Nature Chemical Biology 11, 38-45, doi:10.1038/nchembio.1689 (2014).

$5 \quad$ Hussain, S. et al. MreB filaments align along greatest principal membrane curvature to orient cell wall synthesis. eLife 7, 1239, doi:10.7554/eLife.32471 (2018).

6 Baba, T. et al. Construction of Escherichia coli K-12 in-frame, single-gene knockout mutants: the Keio collection. Molecular Systems Biology 2, 473-411, doi:10.1038/msb4100050 (2006).

7 Mann, P. A. et al. Murgocil is a Highly Bioactive Staphylococcal-Specific Inhibitor of the Peptidoglycan Glycosyltransferase Enzyme MurG. ACS Chemical Biology 8, 24422451, doi:10.1021/cb400487f (2013).

8 Joelsson, A., Liu, Z. \& Zhu, J. Genetic and phenotypic diversity of quorum-sensing systems in clinical and environmental isolates of Vibrio cholerae. Infection and immunity 74, 1141-1147, doi:10.1128/IAI.74.2.1141-1147.2006 (2006).

9 Metcalf, W. W. et al. Conditionally Replicative and Conjugative Plasmids CarryinglacZ $\alpha$ for Cloning, Mutagenesis, and Allele Replacement in Bacteria. Plasmid 35, 1-13, doi:10.1006/plas.1996.0001 (1996).

10 Khan, S. R., Gaines, J., Roop, R. M. \& Farrand, S. K. Broad-host-range expression vectors with tightly regulated promoters and their use to examine the influence of TraR and TraM expression on Ti plasmid quorum sensing. Applied and Environmental Microbiology 74, 5053-5062, doi:10.1128/AEM.01098-08 (2008).

11 Schroeder, J. W. \& Simmons, L. A. Complete Genome Sequence of Bacillus subtilis Strain PY79. Genome Announc 1, e01085-01013, doi:10.1128/genomeA.01085-13 (2013).

12 Branda, S. S., Gonzalez-Pastor, J. E., Ben-Yehuda, S., Losick, R. \& Kolter, R. Fruiting body formation by Bacillus subtilis. Proc. Natl. Acad. Sci. USA 98, 11621-11626, doi:10.1073/pnas.191384198 (2001).

13 Chai, Y., Beauregard, P. B., Vlamakis, H., Losick, R. \& Kolter, R. Galactose Metabolism Plays a Crucial Role in Biofilm Formation by Bacillus subtilis. mBio 3, e00184-00112e00184-00112, doi:10.1128/mBio.00184-12 (2012). 